\title{
MAPPING FOR ELLIPTIC EQUATIONS
}

BY

\section{J. J. GERGEN AND F. G. DRESSEL}

1. Introduction. We consider in this paper the problem of extending the Riemann mapping theorem to equations of the form

$$
\alpha u_{x}+\beta u_{y}=v_{y}, \quad \gamma u_{x}+\delta u_{y}=-v_{x}
$$

where the coefficients $\alpha, \beta, \gamma, \delta$ are real functions of $(x, y)$ satisfying $0<\alpha$, $0<\alpha \delta-(\beta+\gamma)^{2} / 4$. The final result obtained is stated in the following theorem.

Theorem I. Let $D^{*}$ and $T^{*}$ be simple, closed, plane Jordan curves with interiors $D$ and $T$. Let $z^{(1)}, z^{(2)}, z^{(3)}$ be distinct points on $D^{*}$; and let $Z^{(1)}, Z^{(2)}$, $Z^{(3)}$ be distinct points on $T^{*}$ in the same order on $T^{*}$ as the points $z^{(1)}, z^{(2)}$, $z^{(3)}$ on $D^{*}$. Let $\alpha, \beta, \gamma, \delta$ be real bounded functions of class $C^{\prime}$ on $D$ such that

$$
0<\alpha, \quad 0<\mu<\alpha \delta-(\beta+\gamma)^{2} / 4
$$

(on $D$ ),

where $\mu$ is a constant independent of $(x, y)$. Then there exists $a 1: 1$ and continuous mapping $X=u(x, y), Y=v(x, y)$ of $D^{c}=D+D^{*}$ into $T^{c}=T+T^{*}$, which carries $D, D^{*}, z^{(1)}, z^{(2)}, z^{(3)}$ into $T, T^{*}, Z^{(1)}, Z^{(2)}, Z^{(3)}$, which is of class $C^{\prime}$ on $D$, and is such that

$$
\begin{aligned}
0 & <u_{x} v_{y}-u_{y} v_{x} & & \text { (on D), } \\
\alpha u_{x}+\beta u_{y} & =v_{y}, \quad \gamma u_{x}+\delta u_{y}=-v_{x} & & \text { (on } D) .
\end{aligned}
$$

Our methods are based on the work of Morrey [10], and of the authors in $[6 ; 7]$. The latter in turn is based on work of Douglas [3]. For convenience we refer to [6] as GD. We do not consider the uniqueness question.

To treat (1.1) we consider equations of the following two forms:

$$
\alpha u_{x}+\beta u_{y}=v_{y}, \quad-\beta u_{x}+\alpha u_{y}=-v_{x},
$$

where $0<\alpha$, and

$$
\alpha u_{x}+\beta u_{y}=v_{y}, \quad \beta u_{x}+\delta u_{y}=-v_{x},
$$

where $0<\alpha, \alpha \delta-\beta^{2}=1$. In connection with (1.5) and (1.6) it is found convenient to introduce for study equations of the form

$$
\sum_{k=1}^{m} p_{j k} u_{x}^{k}=v_{j y}, \quad \sum_{k=1}^{m} p_{j k} u_{y}^{k}=-v_{j x} .
$$

Part I below is concerned with equations of this latter type. In Part II we

Presented to the Society, April 24, 1953; received by the editors April 7, 1953. 
use the results of Part I and a result of Morrey [10] to obtain an extension of the Riemann theorem for the equations (1.5). The result obtained is a generalization of the result obtained in GD for equations $p u_{x}=v_{y}, p u_{y}=-v_{x}$, $0<p$. In Part III we consider the equations (1.6) and (1.1). Equations of the type (1.6) for the case of analytic coefficients and for the case of bounded measurable coefficients are treated by Morrey [10]. Theorem I is established in Part III on the basis of results given for (1.5) and (1.6). For references to related work, see $[1 ; 2 ; \mathrm{GD}]$.

2. A lemma. We use complex variable notation. We write

$$
z=x+i y=r e^{i \theta}, \quad \zeta=\xi+i \eta=\rho e^{i \phi}, \quad Z=X+i Y,
$$

where $x, y, r, \theta, \cdots, Y$ are real and $0 \leqq r, \rho$. We write also

$$
u(z)=u(x, y), \quad u_{x}(z)=u_{x}(x, y), \quad \text { etc. }
$$

We fix $a$ as a positive number, and denote by $S$ the interior of the circle $S^{*}$ of radius $a$ about the origin as center. In general we use ${ }^{*}$ for boundary and ${ }^{\circ}$ for closure. We introduce for convenience

Definition 1. A function $W(z)$ (real or complex) will be said to be of class $\varepsilon$ on an open set $D$ if (i) it is continuous on $D$, and if (ii) there corresponds to $W$ a second function $w(z)$ such that, if $R$ is any open rectangle (sides parallel to axes) lying with boundary $R^{*}$ in $D$, then $w \in \mathcal{L}(R)$ and $\int_{R^{*}} W(z) d z$ $=i \iint_{R} z(z) d x d y$, where the integral on $R^{*}$ is taken in the positive sense. If $W \in \mathcal{E}(D)$, then we define

$$
W_{*}(z)=\lim _{h \rightarrow 0_{+}} \frac{1}{i h^{2}} \int_{R_{h^{*}(z)}} W(\zeta) d \zeta,
$$

where $R_{h}^{*}(z)$ is the square with vertices at $z, z+h, z+h+i h, z+i h$, wherever this limit exists.

In accordance with this definition, if $W \in \mathcal{E}(D)$, then $W_{*}$ exists p.p. in $D$, and we have

$$
\int_{R^{*}} W(z) d z=i \iint_{R} W_{*}(z) d x d y
$$

for every open rectangle $R$ such that $R^{c} \subset D$.

We observe that, if $W=U+i V$, where $U$ and $V$ are real, is of class $C^{\prime}$ on an open set $D$, then $W \in \mathcal{E}(D)$ with $W_{*}$ given everywhere in $D$ by

$$
W_{*}=W_{x}+i W_{y}=U_{x}-V_{y}+i\left(U_{y}+V_{x}\right) .
$$

In case $U$ and $V$ are A.C.T. (absolutely continuous in the Tonelli sense, Morrey [10, p. 128]; Tonelli [11]) on $D$, then $W \in \mathcal{E}(D)$ and $W_{*}$ is given by (2.2) p.p. in $D$. Real functions $W$ for which (ii) holds are considered by Evans $[4 ; 5]$. 
The following lemma is basic for our analysis. The lemma is an extension of one given by the authors in GD.

LEMMA 1. Let $W=U+i V \in \mathcal{E}(S)$; and suppose that corresponding to each $t, 0<t<a$, there are positive constants $A=A(t)$ and $\tau=\tau(t)$ such that, if $|z| \leqq t$ and $0<h$, then

$$
\iint_{\sigma(h, z) \cdot S}\left|W_{*}\right| d S \leqq A h^{1+\tau},
$$

where $\sigma(h, z)$ is the interior of the circle of radius $h$ about $z$ as center. Put

$$
\Phi^{(t)}(z)=\frac{1}{2 \pi t} \int_{|\zeta|=t} U(\zeta) d s_{\zeta}+\frac{i}{2 \pi t} \int_{|\zeta|=t} \frac{\zeta+z}{\zeta-z} V(\zeta) d s_{\zeta}
$$

for $|z|<t<a$. Then $\lim _{t \rightarrow a} \Phi^{(t)}(z)=\Phi(z)$ exists for $z$ on $S$. The function $\Phi$ is analytic on $S$, and we have

$$
W(z)=\Phi(z)+\iint_{S}\left[K_{1}(z, \zeta) W_{*}(\zeta)+K_{2}(z, \zeta) \bar{W}_{*}(\zeta)\right] d S_{\zeta},
$$

where $\bar{W}_{*}$ is the conjugate of $W_{*}$, and

$$
K_{1}(z, \zeta)=1 /[2 \pi(z-\zeta)], \quad K_{2}(z, \zeta)=z /\left[2 \pi\left(a^{2}-z \xi\right)\right] .
$$

Proof. The proof follows the reasoning of [GD, pp. 188-189]. For a fixed $t, 0<t<a$, put

$$
\begin{aligned}
H^{(t)}(z) & =W(z)-G^{(t)}(z) \\
& =W(z)-\frac{1}{2 \pi} \iint_{S_{t}}\left[\frac{W_{*}(\zeta)}{z-\zeta}+\frac{z \bar{W}_{*}(\zeta)}{t^{2}-z \xi}\right] d S_{\zeta} \quad(|z| \leqq t),
\end{aligned}
$$

where $S_{t}=\sigma(t, 0)$. The condition (2.3) implies that there are positive constants $A$ and $\tau$ such that

$$
\iint_{\sigma(h, z) \cdot S t^{e}} \frac{\left|W_{*}(\zeta)\right|}{|z-\zeta|} d S_{\zeta} \leqq A h^{r} \quad(0<h,|z| \leqq t) .
$$

It follows that $G^{(t)}$, and accordingly $H^{(t)}$, is continuous on $S_{t}^{e}$. Let $R$ be any open rectangle such that $R^{c} \subset S_{t}$. Making use of (2.1) we obtain

$$
\begin{aligned}
\int_{R^{*}} H^{(t)} d z= & \int_{R^{*}} W d z \\
& -\frac{1}{2 \pi} \iint_{|\zeta|<\iota}\left[W_{*}(\zeta) \int_{R^{*}} \frac{d z}{z-\zeta}+\bar{W}_{*}(\zeta) \int_{R^{*}} \frac{z d z}{t^{2}-z \xi}\right] d S_{\zeta} \\
= & \int_{R^{*}} W d z-i \iint_{R} W_{*} d S=0 .
\end{aligned}
$$


Thus $H^{(t)}$ is analytic in $|z|<t$. We have also $\Im\left[G^{(t)}\right] \equiv 0$ on $|z|=t$. Hence $H^{(t)}-\Phi^{(t)}=B_{0}$ on $|z|<t$, where $B_{0}$ is a real constant. Reasoning as in [GD, p. 189] we find that $B_{0}=0$. Hence $W=\Phi^{(t)}+G^{(t)}$ in $|z|<t$. Comparing $G^{(t)}$ with the integral on the right in (2.4) we find that our conclusions hold.

\section{PART I}

3. Sets $P$. We assume in this part that $m$ is a positive integer and, except in Theorem 9 , that $p_{j k}(x, y)(j, k=1,2, \cdots, m)$ are functions having the following properties.

(3.1) $p_{j k}$ is real and continuous on $S^{c}$, and has bounded, continuous first partial derivatives on $S$.

(3.2) $p_{j k}=p_{k j}$ on $S^{c}$.

(3.3) The form $p_{j k} x^{i} x^{k}$ is positive definite for each $(x, y)$ on $S^{c}$.

In (3.1) and (3.2), $j$ and $k$ are free indices with values from 1 to $m$. In (3.3) they are dummy indices indicating summation from 1 to $m$. These conventions will be used throughout for $j, k$. We use the following:

Definition 2. If $u^{i}$ is real and of class $C^{\prime}$ on $S$, and if corresponding to $\left\{u^{j}\right\}_{1}^{m}$ there is a second sequence $\left\{v_{j}\right\}_{1}^{m}$ of real functions which are of class $C^{\prime}$ on $S$ and which with $\left\{u^{j}\right\}_{1}^{m}$ satisfy

$$
p_{j k} u_{x}^{k}=v_{j y}, \quad p_{j k} u_{y}^{k}=-v_{j x}
$$

on $S$, we shall say that the sequence $\left\{u^{j}\right\}_{1}^{m}$ is of class $P\left(p_{j k}\right)$ and write $\left\{u^{i}\right\}$ $\in \mathcal{P}\left(p_{j k}\right)$, or $\left\{u^{i}\right\} \in \mathcal{P}$. If $u^{i} \in C^{0}\left(S^{c}\right)$ and $\left\{u^{i}\right\} \in \mathcal{P}$, we write $\left\{u^{i}\right\} \in \mathcal{P}^{c}$.

The case $m=1$ is the $p$-harmonic case considered in GD. Our results in this part are concerned largely with generalizations of the properties of $p$ harmonic functions to sets $\mathscr{P}$.

We observe that under the conditions imposed on the $p_{j k}$, the transformation $p_{j k} x^{k}=x_{j}$ has an inverse $x^{j}=q^{j k} x_{k}$ for each $(x, y)$ on $S^{c}$, the coefficients $q^{j k}$ likewise satisfying (3.1), (3.2), (3.3). We note also that there are positive constants $A_{1}, A_{2}$, such that

$$
A_{1} \sum_{j=1}^{m}\left(x^{j}\right)^{2} \leqq p_{j k} x^{j} x^{k} \leqq A_{2} \sum_{j=1}^{m}\left(x^{j}\right)^{2}
$$

for $(x, y)$ on $S^{c}$ and all $x^{i}$ ( $x^{i}$ real).

4. Integral formula and first boundary-value problem. In this article we derive the condition (4.2) for sets $P$, and consider the first boundary-value problem for such sets. If $f$ is real and continuous on $S^{*}$, then $H_{f}$ denotes the function determined by the following conditions:

(4.1) $H_{f} \in C^{0}\left(S^{c}\right) . H_{f}$ is harmonic in $S . H_{f}=f$ on $S^{*}$.

Theorem 1 (Integral formula). If $\left\{u^{j}\right\} \in \mathcal{P}^{c}$, then 


$$
p_{j k}(z) u^{k}(z)=h_{j}(z)+\frac{1}{2 \pi} \iint_{S}\left[p_{j k \xi}(\zeta) g_{\xi}(z, \zeta)+p_{j k \eta} g_{\eta}(z, \zeta)\right] u^{k}(\zeta) d S_{\zeta}
$$

$$
p_{j k} u_{x}^{k}=h_{j x}-\psi_{j k x} u^{k}+\frac{1}{2 \pi} \iint_{S}\left[p_{j k \xi g_{\xi x}}+p_{j k \eta} g_{\eta x}\right]\left[u^{k}\right]_{z}^{\zeta} d S_{\zeta}
$$

$(z \in S)$,

where $h_{j}=H_{p_{j k} \boldsymbol{k}^{k}}, \psi_{j k}=H_{p_{j k}}$, and $g$ is Green's function for $S$.

Proof. Let $\left\{v_{j}\right\}_{1}^{m}$ correspond to $\left\{u^{i}\right\}_{1}^{m}$ in the sense of Definition 2. Then $W=-v_{j}+i p_{j k} u^{k} \in C^{\prime}(S)$, and we have, from (3.4), $W_{*}=i p_{j k *} u^{k}$, which is bounded on $S$. Making use of the continuity of $u^{i}$ on $S^{c}$, and applying Lemma 1 , we get

$$
-v_{j}+i p_{j k} u^{k}=\Phi_{i}+i \iint_{S}\left[K_{1} p_{j k *}-K_{2} \bar{p}_{j k *}\right] u^{k} d S_{\zeta}
$$

for $z \in S$, where $\Phi_{j}$ is analytic and $J\left[\Phi_{j}\right]=h_{j}$ on $S$. From (4.4) we get (4.2) for $z \in S$, since $g_{\xi}=2 \pi R\left[K_{1}-K_{2}\right], g_{\eta}=-2 \pi \jmath\left[K_{1}+K_{2}\right]$. Using $g_{\xi}=g_{\eta}=0$ for $z \in S^{*}, \zeta \in S$, we get (4.2) for $z \in S^{*}$. From (4.2) we obtain

$$
\left|\left[p_{j k} u^{k}-h_{j}\right]_{z_{1}}^{z_{2}}\right| \leqq A\left|z_{1}-z_{2}\right| \log 3 a /\left|z_{1}-z_{2}\right| \quad\left(z_{1}, z_{2} \in S^{c}\right),
$$

where $A$ is independent of $z_{1}, z_{2}$. [In inequalities such as (4.5) when one or both members fail to be defined for exceptional values of the variables, we agree that the inequality holds for the values of the variables prescribed for which both members are defined.] We infer that $u^{i}$ satisfies a uniform Hölder condition on each closed subset of $S$. This, and the relation

$$
i p_{j k}(z)=\Psi_{j k}(z)+i \iint_{S}\left[p_{j k *}(\zeta) K_{1}(z, \zeta)-p_{j k *}(\zeta) K_{2}(z, \zeta)\right] d S_{\zeta} \quad(z \in S),
$$

where $\Psi_{j k}$ is analytic and $J\left[\Psi_{j k}\right]=\psi_{j k}$ on $S$, lead to (4.3).

TheOREM 2 (SUfFICIENCY of (4.2)). If $u^{j}$ is real, continuous and bounded on $S$, and if

$$
p_{j k} u^{k}=h_{j}+\frac{1}{2 \pi} \iint_{S}\left[p_{j k \xi g_{\xi}}+p_{j k_{\eta} g_{\eta}}\right] u^{k} d S_{\zeta}
$$

for $z \in S$, where $h_{j}$ is harmonic in $S$ and continuous on $S^{e}$, then $\left\{u^{i}\right\} \in \mathcal{P}^{e}$ when $u^{j}$ is defined on $S^{*}$ as $u^{j}=q^{j k} h_{k}$.

Proof. We can define $v_{j}$ by (4.4) with $\Phi_{j}$ analytic in $S, R\left[\Phi_{j}(0)\right]=0$, say, and $\mathfrak{J}\left[\Phi_{j}\right]=h_{j}$ on $S$. Using (4.6) we find that $u^{j}$ satisfies a uniform Hölder 
condition on each closed subset of $S$. It follows readily that $u^{j}, v_{j} \in C^{\prime}(S)$. For $z$ in $S$ we have

$$
-v_{j x}+i p_{j k} u_{x}^{k}=\Phi_{j x}-\Psi_{j k x} u^{k}+i \iint_{S}\left[K_{1 x} p_{j k *}-K_{2 x} \bar{p}_{j k *}\right]\left[u^{k}\right]_{z}^{\zeta} d S_{\zeta}
$$

These relations and the corresponding ones for the $y$-derivatives give (3.4) in $S$. The conclusion of our theorem then follows on making use of the boundedness of the $u^{j}$ on $S$, and the relationship of the $q^{j k}$ to the $p_{j k}$.

TheOREM 3 (FIRST Boundary-VAlue PROBLEM). Given $f^{i}$ real and continuous on $S^{*}$. There exists one and only one set of functions $\left\{u^{j}\right\}_{1}^{m}$, satisfying

$$
\left\{u^{j}\right\} \in \Phi^{c} ; \quad u^{j}=f^{j} \text { on } S^{*} \text {. }
$$

These functions are given by

$$
u^{j}(z)=h^{j}(z)+\iint_{S} N_{k}^{j}(z, \zeta) h^{k}(\zeta) d S_{\zeta}
$$

for $z \in S$, where $h^{j}=q^{j k} h_{k}, h_{j}=H_{p_{j k^{k}}}$ and the $N_{k}^{j}$ are (i) independent of the $f^{j}$, are (ii) real and continuous for $z, \zeta \in S, z \neq \zeta$, and (iii) satisfy

$$
\left|N_{k}^{j}(z, \zeta)\right| \leqq A /|z-\zeta| \quad(z, \zeta \in S)
$$

We use a special case of the following lemma for the proof of the uniqueness part.

Lemma 2. Let $w^{j} \in C^{\prime}(S)$, and let $\left\{u^{j}\right\} \in \mathcal{P}$. Write

$$
J^{(t)}\left[w^{j}\right]=\iint_{|z|<t} p_{j k} \nabla w^{j} \cdot \nabla w^{k} d S \quad(0<t \leqq a) .
$$

Then

$$
\begin{array}{r}
J^{(t)}\left[w^{j}-u^{j}\right]+J^{(t)}\left[u^{j}\right]=J^{(t)}\left[w^{j}\right]+2 \int_{|z|=t} p_{j k}\left(u^{j}-w^{j}\right) u_{r}^{k} d s \\
\quad(0<t<a) .
\end{array}
$$

Proof of Lemma 2. Denoting by $\left\{v_{j}\right\}_{1}^{m}$ the set corresponding to $\left\{u^{j}\right\}_{1}^{m}$, we have

$$
p_{j k} u_{r}^{k}=\frac{1}{r} v_{j \theta}, \quad p_{j k} u_{\theta}^{k} / r=-v_{j r} \quad(0<|z|<a) .
$$

These relations give

$$
\begin{aligned}
J^{(t)}\left[w^{j}-u^{j}\right]+J^{(t)}\left[u^{j}\right] & \\
& =J^{(t)}\left[w^{j}\right]-2 \iint_{|z|<t} \frac{1}{r}\left[\left(w_{r}^{j}-u_{r}^{j}\right) v_{j \theta}-\left(w_{\theta}^{j}-u_{\theta}^{j}\right) v_{j r}\right] d S
\end{aligned}
$$


for $0<t<a$. Now, if $w \in C^{\prime}$ on $|z| \leqq t$ and $v \in C^{\prime \prime}$ on $|z| \leqq t$, then

$$
\iint_{|z|<t} \frac{1}{r}\left[w_{r} v_{\theta}-w_{\theta} v_{r}\right] d S=\frac{1}{t} \int_{|z|-t} w v_{\theta} d s,
$$

as may be seen by two integrations by parts. Further, by approximating to $v$ with functions of class $C^{\prime \prime}$, this equality is seen to hold also for $v, w \in C^{\prime}$ on $|z| \leqq t$. We obtain then

$$
J^{(t)}\left[w^{i}-u^{i}\right]+J^{(t)}\left[u^{i}\right]=J^{(t)}\left[w^{i}\right]-\frac{2}{t} \int_{|z|-t}\left(w^{i}-u^{j}\right) v_{j \theta} d s,
$$

which with (4.11) gives (4.10).

Proof of uniqueness part of Theorem 3. To prove the uniqueness part of Theorem 3 it suffices to show that, if $u^{i}=0$ on $S^{*}$ and $\left\{u^{i}\right\} \in \mathcal{P}^{c}$, then $u^{i}=0$ on $S$. Now under these hypotheses, $u^{i}$ satisfies (4.5) on $S^{c}$ (with $\left.h_{j} \equiv 0\right)$, whence [(4.2) and (4.3)]

$$
\begin{array}{ll}
\max _{|z|=t}\left|u^{i}(z)\right|=O(\tau \log 3 a / \tau) & (t \rightarrow a), \\
\max _{|z|=t}\left|\nabla u^{i}\right|=O(\log 3 a / \tau) & (t \rightarrow a),
\end{array}
$$

where $\tau=a-t$. Applying Lemma 2 for the case $w^{i} \equiv 0$, we then get

$$
J^{(t)}\left[u^{i}\right]=O\left(\tau \log ^{2} 3 a / \tau\right)=o(1) \quad(t \rightarrow a) .
$$

This gives $J^{(a)}\left[u^{i}\right]=0$, and accordingly $u^{1}=u^{2}=\cdots=u^{m}=0$ on $S$.

Existence and representation. We consider the system of integral equations

$$
u^{i}(z)=h^{i}(z)+\lambda \iint_{S} L_{k}^{i}(z, \zeta) u^{k}(\zeta) d S_{\zeta}
$$

where the $h^{i}$ are arbitrary known functions, real, bounded and continuous on $S$, and

$$
L_{k}^{j}(z, \zeta)=\frac{1}{2 \pi} q^{j k^{\prime}}(z)\left[p_{k k^{\prime} \xi}(\zeta) g_{\xi}(z, \zeta)+p_{k k^{\prime} \eta}(\zeta) g_{\eta}(z, \zeta)\right] \quad \text { (k' dummy). }
$$

Put $z^{n}=\zeta_{n}=3 a n(n=0, \pm 1, \cdots, \pm m)$. Denote by $R^{i}$ the open disc $\left|z-z^{i}\right|$ $<a$, and put $R=R^{1}+\cdots+R^{m}$. The system (4.12) then is equivalent to the system $(c f .[8, \mathrm{pp} .359-360])$

$$
u(z)=h(z)+\lambda \iint_{R} L(z, \zeta) u(\zeta) d R_{\zeta} \quad(z \in R)
$$

under the relations

$$
\begin{array}{cr}
h(z)=h^{j}\left(z-z^{j}\right), \quad u(z)=u^{j}\left(z-z^{j}\right) & \left(z \in R^{j}\right), \\
L(z, \zeta)=L_{k}^{i}\left(z-z^{j}, \zeta-\zeta_{k}\right) & \left(z \in R^{j}, \zeta \in R^{k}\right) .
\end{array}
$$


Now the function $L(z, \zeta)$ is real and continuous for $z, \zeta \in R, z-\zeta \neq z^{n}$ $(n=0, \pm 1, \cdots, \pm(m-1))$ and satisfies

$$
|L(z, \zeta)| \leqq A \sum_{-m+1}^{m-1} 1 /\left|z-\zeta-z^{n}\right| \quad(z, \zeta \in R) .
$$

We know in addition [Theorem 2 and uniqueness] that the system

$$
u(z)=\iint_{R} L(z, \zeta) u(\zeta) d R_{\zeta}
$$

possesses no solution, other than the trivial one, which is real, bounded, and continuous on $R$. Put $L_{0}=L$ and

$$
L_{\mu}(z, \zeta)=\iint_{R} L_{\mu-1}\left(z, \zeta^{\prime}\right) L_{0}\left(\zeta^{\prime}, \zeta\right) d R_{\zeta^{\prime}} \quad(\mu=1,2, \cdots) .
$$

We find that $L_{1}$ is continuous for $z, \zeta \in R, z-\zeta \neq z^{n}(n=0, \pm 1, \cdots, \pm(m-1))$, that

$$
\left|L_{1}(z, \zeta)\right| \leqq A \sum_{-m+1}^{m-1} \log 3 a m /\left|z-\zeta-z^{n}\right|,
$$

and that the iterated kernels $L_{2}, L_{3}, \cdots$ are bounded and continuous for $z, \zeta \in R$. In accordance with the account of the Fredholm theory given by Kellogg [9, pp. 287-291, 294, 299-308], it then follows that the system (4.12) for $\lambda=1$ has a unique solution which is real, bounded, and continuous on $R$. This solution can be written

$$
u(z)=h(z)+\iint_{R} N(z, \zeta) h(\zeta) d R_{\zeta} \quad(z \in R),
$$

where the resolvent $N$ depends only upon $L$, is real and continuous for $z, \zeta \in R, z-\zeta \neq z^{n}(n=0, \pm 1, \cdots, \pm(m-1))$, and satisfies (4.16). Put

$$
N_{k}^{j}(z, \zeta)=N\left(z+z^{j}, \zeta+\zeta_{k}\right)
$$

Then $N_{k}^{j}$ depends only upon the $L_{k}^{j}$ and satisfies (ii) and (iii). Further, under (4.14), (4.17) is equivalent to

$$
u^{i}(z)=h^{i}(z)+\iint_{S} N_{k}^{j}(z, \zeta) h^{k}(\zeta) d S_{\zeta}
$$

We accordingly have shown that subject (only) to the condition that $h^{i}$ is real, bounded and continuous on $S$, the system (4.12) for $\lambda=1$ has a unique solution $u^{1}, u^{2}, \cdots, u^{m}$, with $u^{j}$ real, bounded, and continuous on $S$, this solution being given by (4.18). Taking the special case $h^{i}=q^{j k} h_{k}, h_{j}=H_{p_{j k} f^{k}}$, our theorem follows. 
5. Maximum value principle and convergence. As an immediate corollary of Theorem 3 we have

Theorem 4 (MAXImum VAlUe PRINCIPLE). There is a constant $A$ (depending only upon the $\left.p_{j k}\right)$ such that, if $\left\{u^{j}\right\} \in \mathcal{P}^{c}$, then

$$
\max _{z \in S^{c}} \max _{j}\left|u^{j}(z)\right| \leqq A \max _{z \in S^{*}} \max _{j}\left|u^{j}(z)\right| \text {. }
$$

For the $p$-harmonic case $(m=1)$ a proof of Theorem 4 is given in [GD, p. 197] which is independent of integral equation theory. The authors have been unable to extend this method to the general case.

The following theorem is important for its application.

Theorem 5 (Convergence). Let the functions $u^{j}, u_{n}^{j}, p_{n j k}(n=1,2, \cdots)$ satisfy the following conditions:

(5.1) For each $n$, the $p_{n j k}$ satisfy (3.1), (3.2), and (3.3).

(5.2) $p_{n j k} \rightarrow p_{j k}$ uniformly on $S^{c}$.

(5.3) $p_{n j k x} \rightarrow p_{j k x}, p_{n j k y} \rightarrow p_{j k y}$ boundedly on $S$.

(5.4) $\left\{u_{n}^{j}\right\} \in \mathcal{P}^{c}\left(p_{n j k}\right),\left\{u^{j}\right\} \in \mathcal{P}^{c}\left(p_{j k}\right)$.

(5.5) $u_{n}^{j} \rightarrow u^{j}$ boundedly on $S^{*}$.

Then

(5.6) $u_{n}^{j} \rightarrow u^{j}$ boundedly on $S$;

(5.7) $u_{n x}^{j} \rightarrow u_{x}^{j}, u_{n y}^{j} \rightarrow u_{y}^{j}$ boundedly on every closed subset of $S$.

The symbol $\rightarrow$ refers to the limiting process $n \rightarrow \infty$.

Proof. We first observe that, if $w^{j}, T^{j}$ are real, bounded, and continuous on $S$, and if

$$
w^{j}(z)=T^{j}(z)+\iint_{S} L_{k}^{j}(z, \zeta) w^{k}(\zeta) d S_{\zeta} \quad(z \in S),
$$

where the $L_{k}^{j}$ are defined by (4.13), then

$$
\left|w^{j}(z)\right| \leqq T^{j}(z)+A \iint_{s} \frac{T(\zeta)}{|z-\zeta|} d S_{\zeta} \quad(z \in S),
$$

where $A$ is independent of $z, w^{j}, T^{j}$ and $T(\zeta)=\max _{j}\left|T^{j}(\zeta)\right|$. This result is a consequence of the representation (4.18) of the solution of (4.12) for $\lambda=1$.

Now define $\psi_{n j k}, q_{n}^{j k}, L_{n k}^{j}$ with reference to $p_{n j k}$ in the same manner as the corresponding functions have been defined with reference to $p_{j k}$. Put $h_{j}$ $=H_{p_{j k} k^{k}}, h^{j}=q^{j k} h_{k}$, and define $h_{n j}, h_{n}^{j}$ similarly. We have for $n$ fixed

$$
\begin{aligned}
u_{n}^{j}(z)=h_{n}^{j}(z)+\iint_{S} L_{n k}^{j}(z, \zeta) u_{n}^{k}(\zeta) d S_{\zeta} & (z \in S), \\
p_{n j k} u_{x}^{k}=h_{n j x}-\psi_{n j k x} u^{k}+\iint_{S} L_{n k x}^{j}\left[u_{n}^{k}(\zeta)-u_{n}^{k}(z)\right] d S_{\zeta} & (z \in S) .
\end{aligned}
$$


Put

$$
\epsilon_{n}=\text { bound } \sup \iint_{S}\left|L_{n k}^{j}(z, \zeta)-L_{k}^{j}(z, \zeta)\right| d S_{\zeta}
$$

where the bound $\sup$ is taken for $j, k=1,2, \cdots, m$ and $z \in S$. As a consequence of the conditions imposed on the $p_{j k}$ and $p_{n j k}$ it is found that $\epsilon_{n} \rightarrow 0$. This is the key to the theorem. To show that the $u_{n}^{j}$ are uniformly bounded on $S$, we write

$$
\begin{aligned}
u_{n}^{i}(z)=h_{n}^{j}(z) & +\iint_{S}\left[L_{n k}^{j}(z, \zeta)-L_{k}^{j}(z, \zeta)\right] u_{n}^{k}(\zeta) d S_{\zeta} \\
& +\iint_{S} L_{k}^{j}(z, \zeta) u_{n}^{k}(\zeta) d S_{\zeta}
\end{aligned}
$$

and put $\delta_{n}=$ bound $\sup _{z \in S, j}\left|u_{n}^{j}(z)\right|$. The sum of the first two terms in (5.11) is then numerically less than $A+\delta_{n} \epsilon_{n}$ where $A$ does not depend on $n$ or $z, z \in S$. Using (5.8) we then get

$$
\delta_{n} \leqq A+\delta_{n} \epsilon_{n}+A\left(A+\delta_{n} \epsilon_{n}\right) \leqq A+A \delta_{n} \epsilon_{n},
$$

where $A$ does not depend on $n$. This gives $\delta_{n}=O(1)$. Accordingly, the $u_{n}^{j}$ are uniformly bounded on $S$. To obtain $u_{n}^{j} \rightarrow u^{i}$, we write

$$
u_{n}^{i}-u^{j}=T_{n}^{i}+\iint_{S} L_{k}^{i}\left[u_{n}^{k}(\zeta)-u^{k}(\zeta)\right] d S_{\zeta}
$$

where $T_{n}^{j}=h_{n}^{j}-h^{j}+\iint_{S}\left[L_{n k}^{j}-L_{k}^{j}\right] u_{n}^{k}(\zeta) d S_{\zeta}(z \in S)$. We see that $T_{n}^{j} \rightarrow 0$ boundedly on $S$. Putting $T_{n}(z)=\max _{j}\left|T_{n}^{\prime}(z)\right|$ and using (5.8) again we get

$$
\left|u_{n}^{i}-u^{i}\right| \leqq T_{n}+A \iint_{S} \frac{T_{n}(\zeta)}{|z-\zeta|} d S_{\zeta} \quad(z \in S),
$$

where $A$ is independent of $z, j, n$. From $T_{n} \rightarrow 0$ boundedly on $S$, we then obtain $u_{n}^{j} \rightarrow u^{i}$ on $S$. It remains to show that (5.7) holds. With the aid of (5.9) and the boundedness of the $u_{n}^{k}$ we find that for any fixed $t, 0<t<a$,

$$
\left|u_{n}^{i}\left(z_{1}\right)-u_{n}^{i}\left(z_{2}\right)\right| \leqq A\left|z_{1}-z_{2}\right| \log 3 a /\left|z_{1}-z_{2}\right| \quad\left(\left|z_{1}\right|,\left|z_{2}\right| \leqq t\right),
$$

where $A$ does not depend on $j, n, z_{1}, z_{2}$. This and $\delta_{n}=O(1)$ give

$$
\begin{aligned}
\iint_{S}\left[p_{n j k \xi g_{\xi x}}+p_{n j k \eta} g_{\eta x}\right]\left[u_{n}^{k}\right]_{z}^{\zeta} d S_{\zeta} & \\
& \rightarrow \iint_{S}\left[p_{j k \xi g_{\xi x}}+p_{i k_{\eta} g_{\eta x}}\right]\left[u^{k}\right]_{z}^{\zeta} d S_{\zeta}
\end{aligned}
$$

boundedly on $|z| \leqq t$. We then obtain (5.7) from (5.10) and (5.12). 
6. Dirichlet and Douglas integrals. We derive next the minimal property of sets $P$ with reference to the (generalized) Dirichlet integral, and the inequalities relating the Dirichlet integral for sets $P$ and the (generalized) Douglas integral.

Definition 3. If $w^{i} \in C^{\prime}(S)$, then

$$
J\left[w^{j}\right]=J\left[w^{i} ; p_{j k}\right]=\iint_{S} p_{j k} \nabla w^{j} \cdot \nabla w^{k} d S
$$

is the (generalized) Dirichlet integral for $\left\{w^{j}\right\}_{1}^{m}$. If $w^{j} \in C^{0}\left(S^{*}\right)$, then

$$
D\left[w^{j}\right]=\frac{1}{2 \pi} \int_{S^{*}} \int_{S^{*}} \sum_{j=1}^{m}\left|\frac{w^{j}\left(z_{1}\right)-w^{j}\left(z_{2}\right)}{z_{1}-z_{2}}\right|^{2} d s_{z_{1}} d s_{z_{2}}
$$

is the (generalized) Douglas integral for $\left\{w^{j}\right\}_{1}^{m}$.

Theorem 6 (Minimum of $J$ ). Suppose that $\left\{u^{i}\right\} \in \mathbb{P}^{c}$, that $w^{i}$ is real, $w^{j} \in C^{0}\left(S^{c}\right), w^{j} \in C^{\prime}(S)$ and that $u^{j}$ and $w^{j}$ coincide on $S^{*}$. Then the Dirichlet integral for $\left\{u^{j}\right\}_{1}^{m}$ does not exceed the Dirichlet integral for $\left\{w^{j}\right\}_{1}^{m}$.

Proof. We show that the conditions prescribed for $u^{i}$ and $w^{i}$ imply

$$
J\left[w^{i}-u^{i}\right]+J\left[u^{i}\right] \leqq J\left[w^{i}\right] .
$$

From (6.1) the conclusion of our theorem follows.

Case I. Suppose first that $p_{j k}$ and $u^{i} \in C^{\prime \prime}\left(S^{*}\right)$. Under these conditions, $h_{j}=H_{p_{i k}{ }^{k}}$ and $\psi_{j k}$ have bounded first partial derivatives on $S$. We find then that $p_{j k} u^{k}$ satisfies (4.5) on $S$. There results that $u^{j}$ satisfies (4.5) on $S$. It then follows that $p_{j k} u_{x}^{k}$ and accordingly $u_{q}^{j}$ is bounded on $S$. Making use of Lemma 2 we get

$$
J\left[w^{j}-u^{i}\right]+J\left[u^{j}\right]=J\left[w^{j}\right]
$$

for this case.

Case II. Suppose next that $w^{j} \in C^{\prime}\left(S^{c}\right)$. We approximate to $p_{j k}$ and $w^{i}$ by sequences $\left\{p_{n j k}\right\}_{1}^{\infty},\left\{w_{n}^{j}\right\}_{1}^{\infty}$ satisfying the following conditions:

$$
\begin{aligned}
& \text { for } p_{j k}:(5.1),(5.2),(5.3) ; p_{n j k} \in C^{\prime \prime}\left(S^{c}\right) ; \\
& \text { for } w^{j}: w_{n}^{j} \in C^{\prime \prime}\left(S^{c}\right) ; w_{n}^{i}, w_{n x}^{j}, w_{n y}^{i} \text { tend to } w^{j}, w_{x}^{j}, w_{y}^{j} \text { boundedly on } S^{c} .
\end{aligned}
$$

Denoting by $u_{n}^{j}$ the functions determined by

$$
\left\{u_{n}^{j}\right\} \in P^{c}\left(p_{n j k}\right), \quad u_{n}^{j}=w_{n}^{j} \text { on } S^{*},
$$

we have by Case I

$$
J\left[w_{n}^{j}-u_{n}^{j} ; p_{n j k}\right]+J\left[u_{n}^{j} ; p_{n j k}\right]=J\left[w_{n}^{j} ; p_{n j k}\right] .
$$

Now 


$$
p_{n j k} \nabla w_{n}^{j} \cdot \nabla w_{n}^{j} \rightarrow p_{j k} \nabla w^{j} \cdot \nabla w^{k}
$$

boundedly on $S$, while

$$
p_{n j k} \nabla\left(w_{n}^{j}-u_{n}^{j}\right) \cdot \nabla\left(w_{n}^{k}-u_{n}^{k}\right), \quad p_{n j k} \nabla u_{n}^{j} \cdot \nabla u_{n}^{k}
$$

are non-negative and tend to

$$
p_{j k} \nabla\left(w^{j}-u^{j}\right) \cdot \nabla\left(w^{k}-u^{k}\right), \quad p_{j k} \nabla u^{j} \cdot \nabla u^{k}
$$

on $S$. Thus, using Fatou's lemma and Case I, we get

$$
\begin{aligned}
J\left[w^{j}-u^{j} ; p_{j k}\right]+J\left[u^{j} ; p_{j k}\right] & \leqq \lim \inf J\left[w_{n}^{j}-u_{n}^{j} ; p_{n j k}\right]+\lim \inf J\left[u_{n}^{j} ; p_{n j k}\right] \\
& \leqq \lim \inf J\left[w_{n}^{j} ; p_{n j k}\right]=J\left[w^{j} ; p_{j k}\right],
\end{aligned}
$$

which is (6.1) for this case.

Case III. Consider now the general case. If $J\left[w^{j}\right]=+\infty$ there is nothing to prove. Suppose then that $J\left[w^{j}\right]<+\infty$. Select $\left\{t_{n}\right\}_{1}^{\infty}$ so that $0<t_{1}<t_{2}$ $<\cdots, t_{n} \rightarrow 1$. Put $w_{n}^{j}(z)=w^{j}\left(t_{n} z\right)$. Then $w_{n}^{j} \in C^{\prime}\left(S^{c}\right)$. Denoting by $u_{n}^{j}$ the functions determined by

$$
\left\{u_{n}^{j}\right\} \in \Phi^{c}, u_{n}^{j}=w_{n}^{j} \text { on } S^{*},
$$

we have as a consequence of Case II,

$$
J\left[w_{n}^{j}-u_{n}^{i}\right]+J\left[u_{n}^{i}\right] \leqq J\left[w_{n}^{j}\right] .
$$

Now, again, the limit inferior of the left-hand member of this inequality is not less than $J\left[w^{i}-u^{j}\right]+J\left[u^{j}\right]$. On the other hand, $J\left[w_{n}^{j}\right]$ can be written

$$
J\left[w_{n}^{j}\right]=\iint_{|z|<t_{n} a} p_{j k}\left(z / t_{n}\right) \nabla w^{j}(z) \cdot \nabla w^{k}(z) d S .
$$

We have for $|z|<t_{n} a$

$$
\begin{aligned}
0 & \leqq p_{j k}\left(z / t_{n}\right) \nabla w^{j}(z) \cdot \nabla w^{k}(z) \\
& \leqq A_{2} \sum_{1}^{m}\left|\nabla w^{j}(z)\right|^{2} \leqq \frac{A_{2}}{A_{1}} p_{j k}(z) \nabla w^{j}(z) \cdot \nabla w^{k}(z),
\end{aligned}
$$

which $\in \mathcal{L}$ on $S$. For each $z$ on $S$ we have also

$$
p_{j k}\left(z / t_{n}\right) \nabla w^{j}(z) \cdot \nabla w^{k}(z) \rightarrow p_{j k}(z) \nabla w^{j}(z) \cdot \nabla w^{k}(z) \text {. }
$$

From Lebesgue's theorem it then follows that $J\left[w_{n}^{j}\right] \rightarrow J\left[w^{i}\right]$. We conclude that (6.1) holds for the general case.

Theorem 7 (Douglas Integral). If $\left\{u^{i}\right\} \in \mathcal{P}^{c}$, then the Douglas and Dirichlet integrals for $\left\{u^{j}\right\}_{1}^{m}$ satisfy

$$
A_{1} D\left[u^{j}\right] \leqq J\left[u^{j}\right] \leqq A_{2} D\left[u^{j}\right]
$$

Proof. Put $h^{j}=H_{u j}$. Then 


$$
\begin{aligned}
A_{1} D\left[u^{j}\right] & =A_{1} \iint_{S} \sum_{j=1}^{m}\left|\nabla h^{j}\right|^{2} d S \leqq A_{1} \iint_{S} \sum_{j=1}^{m}\left|\nabla u^{j}\right|^{2} d S \leqq J\left[u^{j}\right], \\
J\left[u^{j}\right] & \leqq \iint_{S} p_{j k} \nabla h^{j} \cdot \nabla h^{k} d S \leqq A_{2} \iint_{S} \sum_{1}^{m}\left|\nabla h^{j}\right|^{2} d S=A_{2} D\left[u^{j}\right] .
\end{aligned}
$$

7. Other conditions on the $p_{j k}$. We conclude this part with two theorems in which other conditions are introduced for the $p_{j k}$.

TheOREM 8 (Solutions of Class $C^{\prime \prime}$ ). If $p_{j k x}, p_{j k y}$ satisfy a uniform Hölder condition on each closed subset of $S$, and if $\left\{u^{j}\right\} \in P$, then $u^{j} \in C^{\prime \prime}(S)$.

Proof. Let $\left\{v_{j}\right\}_{1}^{m}$ correspond to $\left\{u^{j}\right\}_{1}^{m}$. We can suppose that $v_{j}, q^{j k} \in C^{\prime}\left(S^{c}\right)$. The function $W=-q^{j k_{v_{k}}}+i u^{j} \in C^{\prime}(S)$, and we have $W_{*}=-q_{*}^{j k} v_{k}$. Applying Lemma 1, we obtain

$$
u^{j}=h^{j}+\frac{1}{2 \pi} \iint_{S}\left[q_{\xi}^{j k} g_{\eta}-q_{\eta}^{j k} g_{\xi}\right] v_{k} d S_{\zeta}
$$

where $h^{j}=H_{u j}$. Now, if $q \in C^{\prime \prime}\left(S^{c}\right), v \in C^{\prime}\left(S^{c}\right)$, two integrations by parts give

$$
\iint_{S}\left[q_{\xi} g_{\eta}-q_{\eta} g_{\xi}\right] v d S_{\zeta}=\iint_{S} g\left[q_{\eta} v_{\xi}-q_{\xi} v_{\eta}\right] d S_{\zeta} .
$$

By approximation, this relation is likewise seen to hold if $q \in C^{\prime}\left(S^{c}\right)$ and $v \in C^{\prime}\left(S^{c}\right)$. Applying this result to (7.1) we then get

$$
u^{j}=h^{j}+\frac{1}{2 \pi} \iint_{S} g\left[q_{\eta}^{j k} v_{k \xi}-q_{\xi}^{j k} v_{k \eta}\right] d S_{\zeta} \quad(z \in S) .
$$

We find then that $u_{x}^{j}, u_{y}^{j}$ satisfy uniform Hölder conditions on each closed subset of $S$. From our Hölder conditions on $p_{j k x}, p_{j k y}$ we then get that $\left[q_{v}^{j k} v_{k x}-q_{x}^{j k} v_{k y}\right]$ satisfies this condition. Accordingly, $u^{j} \in C^{\prime \prime}(S)$.

Theorem 9 (WeAker conditions for $C^{\prime}$ solutions). Suppose that $p_{j k}$, $u^{j}, v_{j}$ satisfy the following conditions.

$$
\begin{gathered}
p_{j k}, u^{j}, v_{j} \text { are real and A.C.T. on } S . \\
\operatorname{det}\left|p_{j k}\right| \neq 0 \text { on } S .
\end{gathered}
$$

(7.4) Corresponding to every $t, 0<t<a$, there are positive constants $A=A(t)$ and $\tau=\tau(t)$ such that

$$
\begin{array}{lr}
\iint_{\sigma(h, z) \cdot S t^{c}}\left|\nabla p_{j k}\right| d S \leqq A h^{1+r} & (0<h,|z| \leqq t) . \\
p_{j k} u_{x}^{k}=v_{j y}, \quad p_{j k} u_{y}^{k}=-v_{j x} & \quad \text { (p.p.on } S) .
\end{array}
$$

Then $u^{j}, v_{j} \in C^{\prime}(S)$ and satisfy (7.5) everywhere on $S$. 
Proof. We can suppose without loss of generality that $u^{j}, v_{j}, p_{j k} \in C^{0}\left(S^{c}\right)$, that det $\left|p_{j k}\right| \neq 0$ on $S^{c}$, and that there is a $\tau, 0<\tau$, such that

$$
\iint_{\sigma(h, z) \cdot S^{c}}\left|\nabla p_{j k}\right| d S \leqq A h^{1+\tau} \quad\left(0<h, z \in S^{c}\right)
$$

where $A$ is independent of $h$ and $z$. Under these conditions on the $p_{j k}$, the transformation $p_{j k} x^{k}=x_{j}$ has an inverse $x^{j}=q^{j k} x_{k}$ for $(x, y) \in S^{c}$, with $q^{j k}$ $\in C^{0}\left(S^{c}\right), q^{j k}$ A.C.T. on $S$, and $q^{j k}$ satisfying (7.6). Consider the functions

$$
W_{j}=p_{j k} u^{k}+i v_{j}, \quad W^{j}=-q^{j v_{v}}+i u^{j} .
$$

These functions are A.C.T. on $S$. For almost all points on $S$, we have

$$
W_{j *}=p_{j k *} u^{k}, \quad W_{*}^{j}=-q_{*}^{j k} v_{k} \text {. }
$$

Thus $W_{j *}, W_{*}^{\jmath}$ satisfy the condition imposed on $W_{*}$ in Lemma 1. Putting $h^{j}=H_{u_{j}}, h_{j}=H_{v_{j}}$ and applying Lemma 1 , we get

$$
\begin{array}{ll}
v_{j}=h_{j}+\frac{1}{2 \pi} \iint_{S}\left[p_{j k \eta} g_{\xi}-p_{j k \xi} g_{\eta}\right] u^{k} d S_{\zeta} & (z \in S), \\
u^{j}=h^{j}-\frac{1}{2 \pi} \iint_{S}\left[q_{\eta}^{j k} g_{\xi}-q_{\xi}^{j k} g_{\eta}\right] v_{k} d S_{\zeta} & (z \in S) .
\end{array}
$$

Now, if $z_{0}$ is a point in $S$, then $u^{i} \equiv u^{j}\left(z_{0}\right), v_{j} \equiv 0$ satisfy the conditions we have imposed on $u^{j}, v_{j}$. Hence

$$
0=\iint_{S}\left[p_{j k \eta} g_{\xi}-p_{j k \xi} g_{\eta}\right] u^{k}\left(z_{0}\right) d S_{\zeta} \quad(z \in S) .
$$

Making use of this relation and (7.7) we obtain

$$
\left[v_{j}\right]_{z_{0}}^{z}=\left[h_{j}\right]_{z_{0}}^{z}+\frac{1}{2 \pi} \iint_{S}\left[p_{j k \eta} g_{\xi}-p_{j k \xi} g_{\eta}\right]_{z_{0}}^{z}\left[u^{k}\right]_{z_{0}}^{\zeta} d S_{\zeta} \quad\left(z, z_{0} \in S\right) .
$$

Similarly,

$$
\left[u^{j}\right]_{z_{0}}^{z}=\left[h^{j}\right]_{z_{0}}^{z}-\frac{1}{2 \pi} \iint_{S}\left[q_{\eta}^{j k} g_{\xi}-q_{\xi}^{j k} g_{\eta}\right]_{z_{0}}^{z}\left[v_{k}\right]_{z_{0}}^{\zeta} d S_{\zeta} \quad\left(z, z_{0} \in S\right) .
$$

Define $B(z)=\max _{j, k} \max \left[\left|\nabla p_{j k}\right|,\left|\nabla q^{j k}\right|\right]$ at the points $z$ in $S$ where $p_{j k x}, p_{j k y}, q_{x}^{j k}, q_{y}^{j k}$ are defined. Then $B$ satisfies (7.6) and we have

$$
\begin{aligned}
\left|\left[v_{i}\right]_{z_{0}}^{z}\right| \leqq & \left|\left[h_{j}\right]_{z_{0}}^{z}\right| \\
& +A\left|z-z_{0}\right| \iint_{S} \frac{B(\zeta)}{|z-\zeta|\left|\zeta-z_{0}\right|} \max _{k}\left|\left[u^{k}\right]_{z_{0}}^{\zeta}\right| d S_{\zeta}
\end{aligned}
$$




$$
\begin{aligned}
\left|\left[u^{j}\right]_{z_{0}}^{z}\right| \leqq & \left|\left[h^{j}\right]_{z_{0}}^{z}\right| \\
& +A\left|z-z_{0}\right| \iint_{S} \frac{B(\zeta)}{|z-\zeta|\left|\zeta-z_{0}\right|} \max _{z}\left|\left[v_{k}\right]_{z_{0}}^{\zeta}\right| d S_{\zeta}
\end{aligned}
$$

for $z, z_{0} \in S$, where $A$ is independent of $j, z, z_{0}$.

Let $t$ be fixed, $0<t<a$. To prove the theorem it is enough to show that $u^{i}, v_{j} \in C^{\prime}$ on $S_{t}$. We can suppose that $0<\tau<1$ and that $1 / \tau$ is not an integer. We put $n_{0}=[1 / \tau]-1$. We have

$$
\iint_{\sigma(h, z)} \frac{B(\zeta)}{|z-\zeta|} d S_{\zeta} \leqq A h^{\tau} \quad(0<h, z \in S),
$$

where $A$ is independent of $h, z$; and

$$
\iint_{S} \frac{B(\zeta)}{|z-\zeta|\left|\zeta-z_{0}\right|^{1-n \tau}} d S_{\zeta} \leqq \frac{A}{\left|z-z_{0}\right|^{1-(n+1) \tau}}, A \quad\left(z, z_{0} \in S\right),
$$

where $A$ is independent of $z, z_{0}$, the former for $n=0,1, \cdots, n_{0}$, and the latter for $n=n_{0}+1$. Since $u^{i} \in C^{0}\left(S^{c}\right)$, we obtain from (7.10),

$$
\left|\left[v_{i}\right]_{z_{0}}^{z}\right| \leqq\left|\left[h_{i}\right]_{z_{0}}^{z}\right|+A\left|z-z_{0}\right|^{\tau} \quad\left(z, z_{0} \in S\right),
$$

where $A$ does not depend on $j, z, z_{0}$. This gives

$$
\left|\left[v_{j}\right]_{z_{0}}^{z}\right| \leqq A\left|z-z_{0}\right|^{\tau} \quad\left(\left|z_{0}\right|<t, z \in S\right) .
$$

Similarly,

$$
\left|\left[u^{j}\right]_{z_{0}}^{z}\right| \leqq A\left|z-z_{0}\right|^{\tau} \quad\left(\left|z_{0}\right|<t, z \in S\right) .
$$

Applying these results, we then get from (7.10), (7.11), (7.13),

$$
\left|\left[v_{i}\right]_{z_{0}}^{z}\right| \leqq A\left|z-z_{0}\right|^{2 \tau}, \quad\left|\left[u^{j}\right]_{z_{0}}^{z}\right| \leqq A\left|z-z_{0}\right|^{2 \tau} \quad\left(\left|z_{0}\right|<t, z \in S\right)
$$

if $n_{0}>0$, and

$$
\left|\left[v_{j}\right]_{z_{0}}^{z}\right| \leqq A\left|z-z_{0}\right|, \quad\left|\left[u^{i}\right]_{z_{0}}^{z}\right| \leqq A\left|z-z_{0}\right| \quad\left(\left|z_{0}\right| \in t, z \in S\right),
$$

if $n_{0}=0$. After $n_{0}+2$ steps of this kind we get (7.14) in all cases.

Now consider (7.8) with $z_{0}$ fixed in $|z|<t$. If we make use of (7.12) and (7.14), then by the usual device of breaking $S$ into $\sigma\left(h, z_{0}\right)$ and $S-\sigma\left(h, z_{0}\right)$, we find that $v_{j x}\left(z_{0}\right)$ exists and is given by

$$
v_{j x}\left(z_{0}\right)=h_{j x}\left(z_{0}\right)+\frac{1}{2 \pi} \iint_{S}\left[p_{j k \eta} g_{\xi x}\left(z_{0}, \zeta\right)-p_{j k \xi g_{\eta x}}\left(z_{0}, \zeta\right)\right]\left[u^{k}\right]_{z_{0}}^{\zeta} d S_{\zeta} .
$$

Again, making use of (7.12) and (7.14), we find that $v_{j x}$ is continuous in $|z|$ $<t$. Similar reasoning holds for $v_{j y}, u_{x}^{j}, u_{y}^{j}$ and the proof is complete. 


\section{PART II}

8. Functions $(\alpha, \beta)$-regular. We consider in this part equations of the form (1.5). Except in Theorem II, we shall suppose that $\alpha, \beta$ satisfy the following conditions:

(8.1) $\alpha, \beta$ are real and of class $C^{0}$ on $S^{c} . \alpha>0$ on $S^{c}$.

(8.2) $\alpha, \beta$ have continuous, bounded first partial derivatives on $S$.

Following our work on $p$-regular functions we introduce for simplicity of argument

Definition 4. The function $F=u+i v$ is $(\alpha, \beta)$-regular on $S$ if it is of class $C^{\prime}$ on $S$, and if

$$
\alpha u_{x}+\beta u_{y}=v_{y}, \quad-\beta u_{x}+\alpha u_{y}=-v_{x}
$$

on $S$. If $F=u+i v$ is $(\alpha, \beta)$-regular on $S$, then $F^{\prime}$ is defined as

$$
F^{\prime}=\alpha^{1 / 2} \frac{\bar{\chi}}{|\chi|}\left(u_{x}-i u_{y}\right)=\frac{i \alpha^{1 / 2}}{|\chi|}\left(v_{x}-i v_{y}\right),
$$

where $\chi=\alpha-i \beta$.

The case $\beta \equiv 0$ is the case of $p$-regular functions considered in GD.

We take $m=2$ and write

$$
p_{11}=\frac{\alpha^{2}+\beta^{2}}{\alpha}, \quad p_{12}=p_{21}=-\frac{\beta}{\alpha}, \quad p_{22}=\frac{1}{\alpha} .
$$

These functions satisfy (3.1), (3.2), (3.3) and, in addition, satisfy

$$
p_{11} p_{22}-p_{12}^{2}=1
$$

We consider with the equations (8.3) the equations

$$
p_{j k} u_{x}^{k}=v_{j y}, \quad p_{j k} u_{y}^{k}=-v_{j x} .
$$

The results of Part I are applicable to (8.5). We observe that $F=u+i v$ is $(\alpha, \beta)$-regular in $S$ if and only if $u^{1}=u, u^{2}=v, v_{1}=v, v_{2}=-u$ is a solution of (8.5) of class $C^{\prime}$ on $S$.

9. Functions in $\mathcal{E}$. As is the case of $p$-regular mapping, our results for equations (1.5) depend upon properties of functions $\in \mathcal{E}$. In this connection we establish

Theorem 10. (a) Let $\left\{u^{j}\right\} \in \mathcal{P}$. Put

$$
\begin{array}{ll}
Q^{j}=\frac{1}{\left(p_{11}\right)^{1 / 2}}\left[p_{11} \bar{u}_{*}^{1}+p_{12} \bar{u}_{*}^{2}+(-1)^{j} i \bar{u}_{*}^{2}\right] & (z \in S), \\
Q=z^{2} Q^{1} Q^{2}=z^{2} p_{j k} \bar{u}_{*}^{j} \bar{u}_{*}^{k} & (z \in S) .
\end{array}
$$

Then $Q^{j}, Q \in \mathcal{E}$ with 


$$
\begin{array}{ll}
Q_{*}^{j}=-\frac{p_{11 *}}{2 p_{11}} \bar{Q}^{j}-(-1)^{i} i R\left[p_{11}\left(\frac{p_{12}}{p_{11}}\right)_{*} Q^{i}\right] & (z \in S), \\
Q_{*}=z^{2}\left[Q_{*}^{1} Q^{2}+Q^{1} Q_{*}^{2}\right]=-z^{2} \bar{p}_{j k *} \nabla u^{j} \cdot \nabla u^{k} & (z \in S) .
\end{array}
$$

(b) Let $F=u+i v$ be $(\alpha, \beta)$-regular in S. Put $G=\chi u+i v$. Then $G$ and $F^{\prime} \in \mathcal{E}$ with

$$
G_{*}=\chi_{*} u, \quad F_{*}^{\prime}=-\frac{p_{11 *}}{2 p_{11}} \bar{F}^{\prime}-i R\left[p_{11}\left(\frac{p_{12}}{p_{11}}\right)_{*} F^{\prime}\right] \quad(z \in S) .
$$

If for $m$ arbitrary we define $Q$ by $Q=z^{2} p_{j k} \bar{u}_{*}^{j} \bar{u}_{*}^{k}$ we can obtain $Q_{*}=-z^{2} \bar{p}_{j k *} \nabla u^{j}$ $\cdot \nabla u^{k}$, under (3.1), (3.2), (3.3), provided $\left\{u^{j}\right\} \in \mathcal{P}$. A difficulty in extending this material to a generalized Plateau problem of $m$ dimensions lies in the fact that generally $Q$ does not belong to $\mathscr{X}(S)$ [see Definition 5] as for the case $m=2$ with $p_{11} p_{22}-p_{12}^{2}=1$.

Proof. (a) Suppose first that $p_{j k} \in C^{\prime \prime}(S)$. Denote by $v_{1}, v_{2}$ functions corresponding to $u^{1}, u^{2}$. Then $u^{j}, v_{j} \in C^{\prime \prime}(S)$, and we have

$$
\begin{aligned}
& Q^{i}=\frac{i}{\left(p_{11}\right)^{1 / 2}}\left[\bar{v}_{1 *}+(-1)^{i} \bar{u}_{*}^{2}\right], \\
& Q_{*}^{i}=-\frac{p_{11 *}}{2 p_{11}} Q^{i}+\frac{i}{\left(p_{11}\right)^{1 / 2}}\left[\nabla^{2} v_{1}+(-1)^{i} \nabla^{2} u^{2}\right] .
\end{aligned}
$$

From (8.5) for $j=1$ we get

$$
\nabla^{2} v_{1}=\frac{p_{11 y}}{p_{11}} v_{1 y}-p_{11}\left(\frac{p_{12}}{p_{11}}\right)_{x} u_{y}^{2}+\frac{p_{11 x}}{p_{11}} v_{1 x}+p_{11}\left(\frac{p_{12}}{p_{11}}\right)_{y} u_{x}^{2} .
$$

From (8.5) we get also $u_{x}^{2}=-p_{12} v_{1 y}+p_{11} v_{2 y}, u_{y}^{2}=p_{12} v_{1 x}-p_{11} v_{2 x}$, which gives

$$
\nabla^{2} u^{2}=\frac{p_{11 y}}{p_{11}} u_{y}^{2}-p_{11}\left(\frac{p_{12}}{p_{11}}\right)_{x} v_{1 y}+\frac{p_{11 x}}{p_{11}} u_{x}^{2}+p_{11}\left(\frac{p_{12}}{p_{11}}\right)_{y} v_{1 x} .
$$

Substituting in (9.3) we then get (9.1). Next, the first relation in (9.2) is obtained directly from $Q=z^{2} Q^{1} Q^{2}$ for the $C^{\prime \prime}$ case. To get the second relation, we proceed as follows:

$$
\begin{aligned}
Q_{*} & =z^{2}\left[p_{j k *} \bar{u}_{*}^{j} \bar{u}_{*}^{k}+2 p_{j k}\left(\nabla^{2} u^{k}\right) \bar{u}_{*}^{j}\right] \\
& =z^{2} \bar{u}_{*}^{j}\left[p_{j k *} \bar{u}_{*}^{k}+2 p_{j k} \nabla^{2} u^{k}\right] \\
& =z^{2} \bar{u}_{*}^{j}\left[p_{j k *} \bar{u}_{*}^{k}-2 p_{j k x} u_{x}^{k}-2 p_{j k y} u_{y}^{k}\right] \\
& =-z^{2} \bar{u}_{*}^{j} \bar{p}_{j k *} u_{*}^{k}=-z^{2} p_{j k *} \nabla u^{j} \cdot \nabla u^{k}
\end{aligned}
$$

For the general case we can suppose that $u^{i} \in C^{0}\left(S^{c}\right)$. We then approximate to the $p_{j k}$ with sequences $\left\{p_{n j k}\right\}$ satisfying $p_{n 11} p_{n 22}-p_{n 12}^{2}=1, p_{n j k}$ 
$\in C^{\prime \prime}(S)$, and (5.1), (5.2), (5.3). Determining $u_{n}^{j}$ by

$$
\left\{u_{n}^{j}\right\} \in P^{c}\left(p_{n j k}\right), u_{n}^{j}=u^{j} \text { on } S^{*},
$$

and denoting by $v_{n 1}, v_{n 2}$ the functions corresponding to $u_{n}^{1}, u_{n}^{2}$, we then have by the $C^{\prime \prime}$ case

$$
\int_{R^{*}} Q_{n}^{j}(z) d z=i \iint_{R} Q_{n *}^{j} d S
$$

for every rectangle $R$ lying with $R^{*}$ in $S$, where $Q_{n}^{j}$ is defined similarly to $Q^{i}$. Letting $n \rightarrow \infty$ we obtain (Theorem 8 )

$$
\int_{R^{*}} Q^{i} d z=i \iint_{R}\left[-\frac{p_{11 *}}{2 p_{11}} \bar{Q}^{i}-(-1)^{i i R}\left[p_{11}\left(\frac{p_{12}}{p_{11}}\right)_{*} Q^{j}\right]\right] d S .
$$

As the integrand on the right here is continuous on $S$, our result follows for the general case for $Q^{i}$. Similar reasoning is applicable for $Q$.

(b) The part relative to $G$ results from $G \in C^{\prime}(S)$, and differentiation. To deal with $F^{\prime}$ we make use of the fact that $u^{1}=u, u^{2}=v, v_{1}=v, v_{2}=-u$ is a solution of (8.5) of class $C^{\prime}$ on $S$. For this solution, $Q^{2}$ reduces to $2 F^{\prime}$.

10. The class $\mathcal{X}$. In GD we considered functions of class $\mathscr{X}$ on $S$. The definition used is equivalent to the following.

Definition 5. A function $W$ is of class $\mathcal{X}$ on $S$ if it is of class $\mathcal{E}$ on $S$, if $W_{*}$ exists at each point of $S$ and is continuous on $S$, and if there is a constant $M$ such that

$$
\left|W_{*}(z)\right| \leqq M|W(z)|
$$

for $z \in S$.

By means of the properties obtained in GD for functions in $\mathcal{X}$ and Theorem 10, certain properties of $(\alpha, \beta)$-regular functions are readily established.

TheOREM 11. Let $F=u+i v$ be $(\alpha, \beta)$-regular on $S$. If $F$ is not identically zero [constant] on $S$, then for every $t, 0<t<a, G=\chi u+i v\left[F^{\prime}\right]$ has at most a finite number of zeros on $|z|<t$, each such zero having a determined multiplicity.

Proof. We have $G \equiv 0$ only when $F^{\prime} \equiv 0$, and $F^{\prime} \equiv 0$ only when $F \equiv$ const. Our conclusions then follow from Theorem 4 of GD and Theorem 10(b) above.

Theorem 12. Let $F$ be $(\alpha, \beta)$-regular in $S$, and of class $C^{0}$ on $S^{c}$. If $F$ is constant on an open arc of $S^{*}$, then $F$ is constant throughout $S^{c}$.

Proof. Write $F=u+i v$. Suppose $u=u_{0}, v=v_{0}$ on an open arc of $S^{*}$. We apply Theorem $5[\mathrm{GD}]$ to $\chi\left(u-u_{0}\right)+i\left(v-v_{0}\right)$.

TheOREM 13. Let $F=u+i v$ be $(\alpha \beta)$-regular in $S$ and of class $C^{0}$ on $S^{c}$, 
Let $Z=X+i Y$ be a constant such that $F \neq Z$ on $S^{*}$. Then the number of zeros of $W=\chi(u-X)+i(v-Y)$ in $S$ is finite. If $N$ is the total number of these zeros, each counted according to its multiplicity, then

$$
N=\frac{1}{2 \pi}[\Delta \operatorname{amp}(F-Z)]_{S^{*}}
$$

where $[\Delta \operatorname{amp}(F-Z)]_{S^{*}}$ is the variation of the amplitude of $(F-Z)$ on $S^{*}$ in the positive sense.

Proof. The first part of this theorem is a consequence of Theorem 11. Next we can write

$$
W=\frac{1}{2}(\chi+1)(F-Z)\left[1+\frac{\chi-1}{\chi+1} \frac{\bar{F}-\bar{Z}}{F-Z}\right]
$$

The second term in the brackets is continuous and less numerically than 1 on $S^{*}$. Hence $[\Delta \text { amp } W]_{S^{*}}=[\Delta \text { amp }(F-Z)]_{s^{*}}$. This gives (10.1) (Lemma $4[\mathrm{GD}])$.

THEOREM 14. Let $F$ be $(\alpha, \beta)$-regular and not constant on $S$. Let $z_{0}$ be a point in $S$. Then there is a positive integer $n=n\left(z_{0}\right)$, and corresponding to each sufficiently small positive number $t$, a positive number $\tau=\tau(t)$ such that, if 0 $<\left|Z-F\left(z_{0}\right)\right|<\tau$, then there are $n$ and only $n$ points in $S$ satisfying $0<\left|z-z_{0}\right|$ $<t, F(z)=Z$. The case $n=1$ corresponds to the case $F^{\prime}\left(z_{0}\right) \neq 0$.

Proof. This proof is word for word the same as the proof of Theorem 19 [GD], if in the latter we replace $p$ by $\chi$ and $p^{2}$ by $|\chi|^{2}$.

TheOREM 15. Let $\left\{u^{j}\right\} \in P$, and suppose that $J\left[u^{j}\right]<+\infty$. If the analytic function $\Phi$ corresponding to $Q=z^{2} p_{j k} \bar{u}_{*}^{j} \bar{u}_{*}^{k}$ in the sense of Lemma 1 has the form

$$
\Phi=B_{0}+i \sum_{n=1}^{3} B_{n} \frac{z+z^{(n)}}{z-z^{(n)}},
$$

where $B_{0}, B_{1}, B_{2}, B_{3}$ are real constants, and $z^{(1)}, z^{(2)}, z^{(3)}$ are points on $S^{*}$, then either $F=u^{1}+i u^{2}$ is $(\alpha, \beta)$-regular on $S$, or $\bar{F}=u^{1}-i u^{2}$ is $(\alpha,-\beta)$-regular on $S$.

Proof. The integral $\iint_{S}|Q| d S$ is seen to be finite. Further, $Q \in \mathcal{A}(S)$. Suppose, if possible, that $Q \not \equiv 0$ on $S$. Then by Theorem 6 [GD], $Q$ has at most a simple zero on $S$. But $Q$ has a zero at $z=0$ of multiplicity at least two. Hence $Q$ vanishes identically on $S$. In the notation of Theorem 10(a), we then have $Q^{1} Q^{2} \equiv 0$ on $S$. We show that $Q^{1}$ or $Q^{2}$ vanishes identically on $S$. Suppose $Q^{1} \neq 0$. Then there is a point $z_{0}$ of $S$ at which $Q^{1} \neq 0$. In the neighborhood of $z_{0}$, we then have $Q^{2} \equiv 0$. But $Q^{2} \in \mathcal{X}(S)$. Hence, by Theorem 4 [GD], $Q^{2} \equiv 0$ on $S$. Now suppose that $Q^{1} \equiv 0$ on $S$. Then

$$
p_{11} u_{x}^{1}+p_{12} u_{x}^{2}=u_{y}^{2}, \quad p_{11} u_{y}^{1}+p_{12} u_{y}^{2}=-u_{x}^{2}
$$


This gives

$$
\alpha u_{x}^{1}+\beta u_{y}^{1}=u_{y}^{2}, \quad-\beta u_{x}^{1}+\alpha u_{y}^{1}=-u_{x}^{2} .
$$

On the other hand, if $Q^{2} \equiv 0$, we obtain

$$
\alpha u_{x}^{1}-\beta u_{y}^{1}=-u_{y}^{2}, \quad \beta u_{x}^{1}+\alpha u_{y}^{1}=u_{x}^{2} .
$$

The theorem follows.

11. First mapping theorem for (1.5). We prove now

TheOREM 16. The conclusions of Theorem 1 hold for the case $S=D$ if $\delta=\alpha$, $\gamma=-\beta$ and $\alpha, \beta$ satisfy (8.1) and (8.2).

The proof of this theorem follows closely the proof of Theorem 1 [GD]. In the present case we use the Riemann mapping theorem for the analytic case to eliminate the condition that $T^{*}$ is rectifiable, as assumed in GD.

We first prove two lemmas.

Lemma 3. Suppose that $u$, $w$ are real, of class $C^{\prime}$ on $S$, and satisfy

$$
\iint_{S}|\nabla u|^{2} d S<+\infty, \quad \iint_{S}|\nabla w|^{2} d S<+\infty .
$$

Let $p, \lambda$ be of class $C^{\prime}$ on $S$ and have bounded first partial derivatives on $S$. For $\in$ real, $\zeta$ on $S$, put

$$
\begin{aligned}
& u^{(\epsilon)}(\zeta)=u\left(\zeta e^{i \epsilon \lambda(\zeta)}\right), \quad w^{(\epsilon)}(\zeta)=w\left(\zeta e^{i \epsilon \lambda(\zeta)}\right), \\
& J(\epsilon)=\iint_{S} p(\zeta) \nabla u^{(\epsilon)}(\zeta) \cdot \nabla w^{(\epsilon)}(\zeta) d S .
\end{aligned}
$$

Then the derivative $J^{\prime}(0)$ exists and is given by

$$
\begin{gathered}
J^{\prime}(0)=\iint_{S}\left[p\left(\frac{u_{\phi} w_{\phi}}{\rho^{2}}-u_{\rho} w_{\rho}\right) \lambda_{\phi}+p\left(u_{\rho} w_{\phi}+u_{\phi} w_{\rho}\right) \lambda_{\rho}\right. \\
\left.-p_{\phi} \lambda \nabla u \cdot \nabla w\right] d S_{\zeta} .
\end{gathered}
$$

This lemma is a generalization of Lemma 8 [GD].

Proof of Lemma 3. For $0<\rho<a, \phi$ arbitrary, write $r=\rho, \theta=\phi+\epsilon \lambda\left(\rho e^{i \theta}\right)$, $\zeta=\rho e^{i \phi}, z=r e^{i \theta}$. Then $u^{(\epsilon)}(\zeta)=u(z), w^{(e)}(\zeta)=w(z)$, and

$$
\begin{aligned}
p(\zeta) \nabla u^{(\epsilon)}(\zeta) \cdot \nabla w^{(e)}(\zeta)= & p(z)[\nabla u(z) \cdot \nabla w(z)]\left[1+\epsilon \lambda_{\phi}(\zeta)\right] \\
& +[p(\zeta)-p(z)][\nabla u(z) \cdot \nabla w(z)]\left[1+\epsilon \lambda_{\phi}(\zeta)\right] \\
& +\epsilon\left\{p(\zeta)\left[u_{\theta} w_{\theta} / r^{2}-u_{r} w_{r}\right] \lambda_{\phi}+p(\zeta)\left[u_{r} w_{\theta}+u_{\theta} w_{r}\right] \lambda_{\rho}\right\} \\
& +\epsilon^{2} u_{\theta} w_{\theta}|\nabla \lambda(\zeta)|{ }^{2} p(\zeta)=E_{1}+E_{2}+E_{3}+E_{4},
\end{aligned}
$$


say. We note that $\left|E_{n}\right| \leqq A\left\{|\nabla u(z)|^{2}+|\nabla w(z)|^{2}\right\}$, where $A$ does not depend on $\epsilon, \phi, \rho$. Thus $J(\epsilon)$ exists for $\epsilon$ sufficiently small. Denoting the integrand on the right in (11.1) by $I(\zeta)$, we have $|I(\zeta)| \leqq A|\nabla u(\zeta)||\nabla w(\zeta)|$ where $A$ does not depend on $\zeta$. Accordingly, $I \in \mathcal{L}(S)$. For $0<t<a, \epsilon \neq 0$ we have

$$
\begin{aligned}
\left|\frac{J(\epsilon)-J(0)}{\epsilon}-\iint_{S} I(\zeta) d S_{\zeta}\right| & \leqq \int_{0}^{t} \rho d \rho \int_{0}^{2 \pi}\left|\sum_{2}^{4} \frac{E_{n}}{\epsilon}-I(\zeta)\right| d \phi \\
& +\int_{t}^{a} \rho d \rho \int_{0}^{2 \pi}\left\{|I(\zeta)|+\sum_{2}^{4}\left|\frac{E_{n}}{\epsilon}\right|\right\} d \phi .
\end{aligned}
$$

For $t$ fixed, the integrand on the right in the first term converges boundedly to 0 as $\epsilon \rightarrow 0$, on $0<|\zeta|<t$. The superior limit of the second term is no larger than

$$
\iint_{t<|\zeta|<a}\left\{|I(\zeta)|+A\left[|\nabla u|^{2}+|\nabla w|^{2}\right]\right\} d S_{\zeta},
$$

where $A$ is independent of $t$. The relation (11.1) then follows.

Lemma 4. Let $\left\{u^{i}\right\} \in P$, and suppose that $J\left[u^{i}\right]<+\infty$. Put $Q=U+i V$ $=z^{2} p_{j k} \bar{u}_{*}^{j} \bar{u}_{*}^{*}$. Under the hypotheses of Lemma 3 for $\lambda$ we have

$$
\begin{gathered}
\iint_{S}\left[p_{j k}\left(\frac{u_{\phi}^{j} u_{\phi}^{k}}{\rho^{2}}-u_{\rho}^{i} u_{\rho}^{k}\right) \lambda_{\phi}+p_{j k}\left(u_{\rho}^{i} u_{\phi}^{k}+u_{\phi}^{j} u_{\rho}^{k}\right) \lambda_{\rho}-p_{j k \phi} \lambda \nabla u^{j} \cdot \nabla u^{k}\right] d S \\
=-\lim _{t \rightarrow a} \frac{1}{t} \int_{|\xi|=t} \lambda V d s .
\end{gathered}
$$

Proof of Lemma 4. Denote by $I(\zeta)$ the integrand in the integral on the left. Then $I \in \mathcal{L}(S)$, and the lemma will be proved if we can show that

$$
\iint_{|\zeta|<t} I(\zeta) d S_{\zeta}=-\frac{1}{t} \int_{|\xi|=t} \lambda V d s_{\zeta} \quad(0<t<a) .
$$

Suppose first that $p_{j k} \in C^{\prime \prime}(S)$. We calculate $U, V$ to obtain

$$
U=p_{j k} r^{2}\left(u_{r}^{i} u_{r}^{k}-u_{\theta}^{i} u_{\theta}^{k} / r^{2}\right), \quad V=-p_{j k} r^{2} \nabla u^{j} \cdot \nabla u^{k} \quad(0<|z|<a) .
$$

Now

$$
\begin{aligned}
Q_{*} & =\frac{z}{r}\left[U_{r}-\frac{1}{r} V_{\theta}+i\left(\frac{1}{r} U_{\theta}+V_{r}\right)\right] \\
& =-z^{2}\left[\frac{\bar{z}}{r}\left(p_{j k r}-i \frac{p_{j k \theta}}{r}\right) \nabla u^{j} \cdot \nabla u^{k}\right] \quad(0<|z|<a),
\end{aligned}
$$

whence 


$$
\frac{1}{r} U_{\theta}+V_{r}=r p_{j k \theta} \nabla u^{i} \cdot \nabla u^{k} \quad(0<|z|<a) .
$$

Thus

$$
\begin{aligned}
\iint_{|\zeta|<t} I d S_{\zeta}= & \iint_{|\zeta|<t}\left[-\frac{U}{\rho^{2}} \lambda_{\phi}-\frac{V}{\rho} \lambda_{\rho}-p_{j k \phi} \lambda \nabla u^{j} \cdot \nabla u^{k}\right] d S_{\zeta} \\
= & -\frac{1}{t} \int_{|\zeta|=t} \lambda V d s \\
& +\iint_{|\zeta|<t}\left[\frac{U_{\phi}}{\rho}+V_{\rho}-\rho p_{j k \phi} \nabla u^{j} \cdot \nabla u^{k}\right] \frac{\lambda}{\rho} d S_{\zeta} .
\end{aligned}
$$

From (11.4) we then obtain (11.3) for the $C^{\prime \prime}$ case. By approximation, (11.3) then follows for the $C^{\prime}$ case. This completes the proof.

Proof of Theorem 16. We can suppose that the $z^{(n)}\left[Z^{(n)}\right]$ are arranged in positive order on $S^{*}\left[T^{*}\right]$, with $z^{(n)}=a e^{i(n)}, 0 \leqq \theta^{(1)}<\theta^{(2)}<\theta^{(3)}<2 \pi$. We put $\theta^{(t)}=\theta^{(1)}+2 \pi$. By the Riemann mapping theorem for the analytic case there exists a function $\omega=\omega^{1}+i \omega^{2}$ which is analytic in $S$, continuous on $S^{c}$, and which maps $S^{c}$ onto $T^{c}$ in a $1: 1$ manner with $S, S^{*}, z^{(n)}$ corresponding to $T, T^{*}, Z^{(n)}$. For $\omega$ we have in particular the following three properties:

$$
\begin{gathered}
\omega\left(a e^{i \theta^{(n)}}\right)=Z^{(n)} \quad(n=1,2,3), \\
\iint_{S}\left|\nabla \omega^{1}\right|^{2} d S=\iint_{S}\left|\nabla \omega^{2}\right|^{2} d S=\operatorname{meas}(T)<+\infty, \\
{[\Delta \operatorname{amp}(\omega-Z)]_{S^{*}}=2 \pi \text { if } Z \in T, 0 \text { if } Z \in \operatorname{comp} T^{c} .}
\end{gathered}
$$

We denote by $\Lambda$ the class of functions $b(\theta)$ having the following properties:

$$
\begin{aligned}
b\left(\theta^{(n)}\right) & =\theta^{(n)} & (n=1,2,3,4), \\
b(\theta+2 \pi) & =b(\theta)+2 \pi & \text { (all } \theta),
\end{aligned}
$$

$$
b(\theta) \text { is continuous and nondecreasing on }(-\infty,+\infty) \text {. }
$$

Let $b \in \Lambda$. Corresponding to $b$ there are functions $u^{1}, u^{2}$ satisfying

$$
\left\{u^{j}\right\} \in \mathcal{P}^{c}, \text { and } u^{i}\left(a e^{i \theta}\right)=\omega^{j}\left(a e^{i b(\theta)}\right)
$$

We put $R[b]=J\left[u^{i}\right]$. The proof of our theorem depends upon minimizing $R$ in $\Lambda$.

We observe first that the lower bound $R_{0}$ for $R[b]$ for $b \in \Lambda$ is finite. Indeed, we need only take $b(\theta)=\theta$. For the corresponding $u^{1}, u^{2}$ we have

$$
J\left[u^{i}\right] \leqq J\left[\omega^{j}\right] \leqq A_{2} \iint_{S}\left\{\left|\nabla \omega^{1}\right|^{2}+\left|\nabla \omega^{2}\right|^{2}\right\} d S=2 A_{2} \text { meas } T<+\infty .
$$


We observe next that there exists a member $b$ of $\Lambda$ for which $R[b]=R_{0}$. In fact, using Helly's theorem we can select a convergent sequence $\left\{b_{n}\right\}$ of members of $\Lambda$ such that

$$
R\left[b_{n}\right]<+\infty, \quad R\left[b_{n}\right] \rightarrow R_{0} .
$$

Put $b(\theta)=\lim b_{n}(\theta)$. Then $b(\theta)$ satisfies (11.8), (11.9), and (11.10), except possibly for the continuity condition. Using Fatou's theorem and Theorem 7 we get

$$
\int_{0}^{2 \pi} d \theta_{1} \int_{0}^{2 \pi} \frac{\left|\omega\left(a e^{i b\left(\theta_{1}\right)}\right)-\omega\left(a e^{i b\left(\theta_{2}\right)}\right)\right|^{2}}{\sin ^{2} \frac{1}{2}\left(\theta_{1}-\theta_{2}\right)} d \theta_{2} \leqq \frac{8 \pi}{A_{1}} R_{0}<+\infty .
$$

Should $b$ have a discontinuity at $\theta_{0}$, say, then at $\theta_{0}, b$ would have left-hand and right-hand limits $b_{-}$and $b_{+}$satisfying $0<b_{+}-b_{-}<2 \pi$. It would then follow that the integral on the left in (11.12) would have the value $+\infty$, contrary to (11.12). We thus have $b \in \Lambda$. By Theorem 5 and Fatou's lemma we have also $R[b] \leqq \lim R\left[b_{n}\right]=R_{0}$. Accordingly, $R[b]=R_{0}$.

Now fix $b$ such that $b \in \Lambda, R[b]=R_{0}$, and denote by $u^{1}, u^{2}$ the functions determined by $b$. Fix $h$ so that

$$
0<h<\frac{1}{3} \min \left(a,\left|z^{(1)}-z^{(2)}\right|,\left|z^{(1)}-z^{(3)}\right|,\left|z^{(2)}-z^{(3)}\right|\right) .
$$

Fix $\psi(x)$ as a real function of class $C^{\prime}$ on $0 \leqq x<+\infty$ such that $\psi(x)=0$ for $0 \leqq x \leqq h, \psi(x)=1$ for $3 h \leqq x$. Put $\Psi^{(n)}(z)=1-\psi\left(\left|z-z^{(n)}\right|\right)$. Then $\Psi^{(n)}$ is of class $C^{\prime}$ in the plane. Put

$$
Q=U+i V=z^{2} p_{j k} \bar{u}_{*}^{i} \bar{u}_{*}^{k}, \quad B_{n}=\lim _{t \rightarrow a} \int_{|\zeta|=t} \Psi^{(n)} V d s_{\zeta} .
$$

Then $B_{n}$ is real. Let $z_{0}$ (fixed for the moment) be such that $\left|z_{0}\right|<h$. Put

$$
\lambda(z)=\lambda\left(z, z_{0}\right)=R\left(\frac{z+z_{0}}{z-z_{0}}\right) \psi(|z|)-\sum_{1}^{3} \Psi^{(n)}(z) R\left(\frac{z^{(n)}+z_{0}}{z^{(n)}-z_{0}}\right) .
$$

Then $\lambda$ is the class $C^{\prime}$ in the plane and vanishes at $z=z^{(n)}$. Put $b^{(e)}(\theta)$ $=b\left(\theta+\epsilon \lambda\left(a e^{i \theta}\right)\right)$. For $\epsilon$ sufficiently small, $|\epsilon|<\epsilon_{0}$, say, $b^{(e)}$ satisfies (11.8), (11.9), (11.10). Put $u^{1(\epsilon)}(\zeta)=u^{1}\left(\zeta e^{i \epsilon \lambda(\zeta)}\right), u^{2(\epsilon)}(\zeta)=u^{2}\left(\zeta e^{i \epsilon \lambda(\zeta)}\right)$, for $|\epsilon|<\epsilon_{0}$. From $R[b]=R_{0}, \quad R_{0} \leqq R\left[b^{(\epsilon)}\right]$, and Theorem 6 we get $J\left[u^{(e)}\right] \geqq R\left[b^{(e)}\right] \geqq R[b]$ $=J\left[u^{i}\right]$. It follows that the derivative $\left[d J\left[u^{i(\epsilon)}\right] / d \epsilon\right]_{\epsilon=0}$, if it exists, must be zero. From $J\left[u^{i}\right]<+\infty$, and Lemma 3 we find that this derivative exists and is given by the left-hand member of (11.2). We infer then from Lemma 4 that

$$
\lim _{t \rightarrow a} \frac{1}{t} \int_{|\zeta|=t} \lambda V d s=0
$$


This gives

$$
\lim _{t \rightarrow a} \frac{1}{2 \pi t} \int_{|\zeta|=t} R\left(\frac{\zeta+z_{0}}{\zeta-z_{0}}\right) V(\zeta) d s_{\zeta}=\sum_{1}^{3} B_{n} R\left(\frac{z^{(n)}+z_{0}}{z^{(n)}-z_{0}}\right) .
$$

If, then, $\Phi$ denotes the analytic function corresponding to $Q$ in the sense of Lemma 1, we have

$$
\Phi\left(z_{0}\right)=B_{0}+i \sum_{1}^{3} B_{n}\left(\frac{z^{(n)}+z_{0}}{z^{(n)}-z_{0}}\right)
$$

where $B_{0}, B_{1}, B_{2}, B_{3}$ are real constants. This result holds for $\left|z_{0}\right|<h$. By analyticity we then have

$$
\Phi(z)=B_{0}+i \sum_{1}^{8} B_{n}\left(\frac{z^{(n)}+z}{z^{(n)}-z}\right)
$$

for $z$ unrestricted. Applying Theorem 15, we then find that either $F=u^{1}+i u^{2}$ is $(\alpha, \beta)$-regular in $S$, or $\bar{F}=u^{1}-i u^{2}$ is $(\alpha,-\beta)$-regular in $S$. These functions $\in C^{0}\left(S^{c}\right)$. Let $Z_{0}=X_{0}+i Y_{0}$ be a point not on $T^{*}$. Then $F \neq Z_{0}$ and $\bar{F} \neq \bar{Z}_{0}$ on $S^{*}$. Let $N\left(Z_{0}\right)$ be the number of zeros of $\chi\left(u^{1}-X_{0}\right)+i\left(u^{2}-Y_{0}\right)$ or of $\bar{\chi}\left(u^{1}-X_{0}\right)$ $-i\left(u^{2}-Y_{0}\right)$ on $S$, according as to whether $F$ is $(\alpha, \beta)$-regular or $\bar{F}$ is $(\alpha,-\beta)$ regular on $S$. We have

$$
\begin{aligned}
& {\left[\Delta \operatorname{amp}\left(F-Z_{0}\right)\right]_{s^{*}}=\left[\Delta \operatorname{amp}\left(\omega-Z_{0}\right)\right]_{s^{*}},} \\
& {\left[\Delta \operatorname{amp}\left(\bar{F}-\bar{Z}_{0}\right)\right]_{s^{*}}=-\left[\Delta \operatorname{amp}\left(\omega-Z_{0}\right)\right]_{s^{*}} .}
\end{aligned}
$$

Suppose, if possible, that $\bar{F}$ is $(\alpha,-\beta)$-regular on $S$. From (11.14) and Theorem 13 we get, if $Z_{0} \in T, N\left(Z_{0}\right)=-1$. It follows that $F$ is $(\alpha, \beta)$-regular on $S$. From (11.13) we have also $N\left(Z_{0}\right)=1$ if $Z_{0} \in T$, and $N\left(Z_{0}\right)=0$ if $Z_{0}$ $\in \operatorname{comp} T^{c}$, for $F$.

We may summarize our results for $F$ so far as follows:

$$
\begin{gathered}
F=u^{1}+i u^{2} \text { is }(\alpha, \beta) \text {-regular in } S . F \in C^{0}\left(S^{c}\right) . \\
F\left(a e^{i \theta}\right)=\omega(b(\theta)), \text { all } \theta . F(S) \subset T^{c} .
\end{gathered}
$$

(11.17) Every point of $T$ is the image of exactly one point on $S$ under $Z=F(z)$.

$$
F\left(z^{(n)}\right)=Z^{(n)} \quad(n=1,2,3) .
$$

Reasoning as in the proof of Theorem 1 [GD] with Theorems 12 and 14 replacing Theorems 17 [GD] and 19 [GD], we get $F(S) \subset T, F^{\prime} \neq 0$ on $S$, and the result that $Z=F(z)$ maps $S^{*}$ onto $T^{*}$ in a $1: 1$ manner. Taking $u=u^{1}$, $v=u^{2}$, and noting that $u_{x} v_{y}-u_{y} v_{x}=\alpha\left|\nabla u^{1}\right|^{2}=\left|F^{\prime}\right|^{2}>0$, we get the conclusions of our theorem.

12. Lightening of conditions. As our final result on equations (1.5) specifically we obtain Theorem I for the special case $D=S, \delta=\alpha, \gamma=-\beta$. This 
result depends upon a theorem of Morrey [10, p. 136].

TheOREM II. The conclusions of Theorem I hold if $D=S, \delta=\alpha, \gamma=-\beta$.

It is convenient to first prove

LEMma 5. Let the functions $F=u+i v, F_{n}=u_{n}+i v_{n}, \alpha, \beta, \alpha, \beta_{n}$ $(n=1,2, \cdots)$ satisfy the following conditions.

(12.1) $\alpha, \beta, \alpha_{n}, \beta_{n}$ satisfy (8.1) and (8.2).

(12.2) $\alpha_{n} \rightarrow \alpha, \beta_{n} \rightarrow \beta$ uniformly on $S^{c}$.

(12.3) $\alpha_{n x} \rightarrow \alpha_{x}, \alpha_{n y} \rightarrow \alpha_{y}, \beta_{n x} \rightarrow \beta_{x}, \beta_{n y} \rightarrow \beta_{y}$ boundedly on $S$.

(12.4) $F_{n}$ is $\left(\alpha_{n}, \beta_{n}\right)$-regular on $S$ and continuous on $S^{c}$.

(12.5) $F_{n} \rightarrow F$ on $S^{c}$, the convergence being uniform on $S^{*}$.

Then $F$ is $(\alpha, \beta)$-regular on $S$.

Proof of Lemma 5. Define $p_{n j k}$ in terms of $\alpha_{n}, \beta_{n}$ by (8.4). Put $u_{n}^{1}=u_{n}$, $u_{n}^{2}=v_{n}, v_{n 1}=v_{n}, v_{n 2}=-u_{n}$. Then $u_{n}^{1}, u_{n}^{2}, v_{n 1}, v_{n 2}$ satisfy $p_{n j k} u_{n x}^{k}=v_{n j y}, p_{n j k} u_{n y}^{k}$ $=-v_{n j x}$ on $S$. Determine $u^{1}, u^{2}$ by $\left\{u^{j}\right\} \in \mathcal{P}^{c}, u_{j}^{1}=u$ on $S^{*}, u^{2}=v$ on $S^{*}$. Then by Theorem 5 we have $u_{n}^{j} \rightarrow u^{j}, u_{n x}^{j} \rightarrow u_{x}^{j}, u_{n y}^{j} \rightarrow u_{y}^{j}$ on $S$. From $v_{n 1}=v_{n}^{2}, u_{n 2}=-u_{n}^{1}$, we then get $v_{n 1 y} \rightarrow u_{y}^{2}, v_{n 2 y} \rightarrow-u_{y}^{1}$. Thus $p_{1 k} u_{x}^{k}=u_{y}^{2}, p_{2 k} u_{x}^{k}=-u_{y}^{1}$ on $S$. On the other hand, as a consequence of (12.5) and $u_{n}^{j} \rightarrow u^{j}$ we get $u=u^{1}, v=u^{2}$. We then have $u, v \in C^{1}$ on $S$ and

$$
\left.\frac{\alpha^{2}+\beta^{2}}{\alpha} u_{x}-\frac{\beta}{\alpha} v_{x}=v_{y}, \quad-\frac{\beta}{\alpha} u_{x}+\frac{1}{\alpha} v_{x}=-u_{y} \quad \text { (on } S\right) .
$$

These give $\alpha u_{x}+\beta u_{y}=v_{y},-\beta u_{x}+\alpha u_{y}=-v_{x}$; and the lemma follows.

Proof of Theorem II. Let $t$ be fixed, $0<t<a$. Select arbitrarily a sequence of numbers $0<a_{1}<a_{2}<\cdots, a_{n} \rightarrow 1$. Put $\alpha_{n}(z)=\alpha\left(a_{n} z\right), \beta_{n}(z)=\beta\left(a_{n} z\right)$. Then $\alpha_{n}, \beta_{n}$ satisfy (8.1) and (8.2) for each $n$. Denote by $u_{n}, v_{n}$ the mapping functions determined by Theorem 16. Then the members of the family of transformations $\Gamma_{n}: X=u_{n}(x, y), Y=v_{n}(x, y)$ have the following properties: (i) $\Gamma_{n}$ carries the Jordan domain $S$, plus boundary $S^{*}$, in a $1: 1$ and continuous manner into the Jordan domain $T$, plus boundary $T^{*}$, with $S, S^{*}, z^{(m)}$ ( $m=1,2,3)$ corresponding to $T, T^{*}, Z^{(m)}$; (ii) $\Gamma_{n}$ is of class $C^{\prime}$ on $S$; (iii) $\left|\nabla u_{n}\right|^{2}+\left|\nabla v_{n}\right|^{2} \leqq A\left(u_{n x} v_{n y}-u_{n y} v_{n x}\right)$ on $S$, where $A$ does not depend on $n$ or $z$. It follows then $\left[10\right.$, p. 136] that the $u_{n}, v_{n}$ are equicontinuous on $S^{c}$, and that if $x=U_{n}(X, Y), y=V_{n}(X, Y)$ denote the inverse of $\Gamma_{n}$, then the $U_{n}, V_{n}$ are equicontinuous on $T^{c}$. As the sequences $u_{n}, v_{n}, U_{n}, V_{n}$ are uniformly bounded on $S^{c}$ and $T^{c}$, there results that we can select integers $l_{1}<l_{2}<\cdots$ such that $\left\{u_{l_{n}}\right\},\left\{v_{l_{n}}\right\},\left\{U_{l_{n}}\right\},\left\{V_{l_{n}}\right\}$ are uniformly convergent on $S^{c}, T^{c}$. Put $u=\lim u_{l_{n}}$, $v=\lim v_{l_{n}}$. We see that $X=u(x, y), Y=v(x, y)$ is a $1: 1$ and continuous mapping of $S^{c}$ into $T^{c}$ with $S, S^{*}, z^{(m)}$ corresponding to $T, T^{*}, Z^{(m)}$. Further if $t$ is fixed, $0<t<a$, then $\alpha, \beta, \alpha_{n}, \beta_{n}, F_{n}=u_{n}+i v_{n}, F=u+i v$ satisfy the conditions of Lemma 5 relative to $|z| \leqq t$. It follows that $F$ is $(\alpha, \beta)$-regular in $|z|<t$, and 
accordingly in $S$. From $u_{x} v_{y}-u_{y} v_{x}=\alpha\left(u_{x}^{2}+u_{y}^{2}\right)$ we get $u_{x} v_{y}-u_{y} v_{x} \geqq 0$. By Theorem 14, the equality cannot hold. Theorem II is then proved.

\section{PART III}

13. Mapping for (1.6). In this part we consider the equations (1.6) and (1.1). Basing our proof on the work of Morrey, we first establish the following theorem for the equations (1.6).

THEOREM III. The conclusions of Theorem I hold for the case $T=S$, and $\beta=\gamma, \alpha \delta-\beta^{2}=1$ on $D$.

Proof. Under our hypotheses, the functions $\alpha, \beta, \delta$ are bounded and measurable on $D$. From $0<\alpha$ and $\alpha \delta-\beta^{2}=1$ it follows from the work of Morrey [10, in particular Theorem 3, p. 141 and Theorem 4, p. 143] that there exists a pair of functions $u(x, y), v(x, y)$ for which the following are true:

(13.1) The transformation $\Gamma: X=u(x, y), Y=v(x, y)$ carries $D^{c}$ into $S^{c}$ in a $1: 1$ manner, with $D, D^{*}, z^{(n)}$ corresponding to $S, S^{*}, Z^{(n)}$.

(13.2) $u, v$ are A.C.T. on $D$, and $|\nabla u|^{2},|\nabla v|^{2}$ are integrable $\mathcal{L}$ over closed subsets of $D$.

(13.3) $U, V$, where $x=U(X, Y), y=V(X, Y)$ is the inverse $\Gamma^{-1}$ of $\Gamma$, are A.C.T. on $S$.

$$
U_{X} V_{Y}-U_{Y} V_{X} \neq 0
$$

p.p. on $S$.

(13.5) If $\Delta$ is a measurable subset of $S$, then $\Gamma^{-1}(\Delta)$ is measurable and meas $\Gamma^{-1}(\Delta)=\iint_{\Delta}\left(U_{X} V_{Y}-U_{Y} V_{X}\right) d S$.

(13.6) If $0<t<a$, then there corresponds to $t$ positive numbers $A=A(t)$, $\tau=\tau(t)$ such that $\left|U(Z)-U\left(Z_{0}\right)\right| \leqq A\left|Z-Z_{0}\right| \tau,\left|V(Z)-V\left(Z_{0}\right)\right| \leqq A\left|Z-Z_{0}\right|^{\tau}$ for $|Z|,\left|Z_{0}\right| \leqq t$.

(13.7) If $w(x, y)$ is A.C.T. on $D,|\nabla w|^{2} \in \mathcal{L}$ on measurable subsets of $D$, and if $W=w(U, V)$, then $W$ is A.C.T. on $S$ and

$$
\begin{array}{ccc}
W_{X}=w_{x} U_{X}+w_{y} V_{X}, & W_{Y}=w_{x} U_{Y}+w_{y} V_{Y} & \text { (p.p. on } S) . \\
\alpha u_{x}+\beta u_{y}=v_{y}, & \beta u_{x}+\delta u_{y}=-v_{x} & \text { (p.p. on } D) .
\end{array}
$$

We prove on the basis of our assumptions on $\alpha, \beta, \delta$, and the properties enumerated above for $u, v, U, V$ that $u, v \in C^{\prime}(D)$ and that $u_{x} v_{y}-u_{y} v_{x}>0$ on $D$. These with (13.1) and (13.8) will complete the proof.

Taking $w=u(x, y)$ and $w=v(x, y)$, and applying (13.2), (13.4), (13.7), and (13.8), we get

$$
p U_{X}+q U_{Y}=V_{Y}, \quad-q U_{X}+p U_{Y}=-V_{X}
$$

p.p. in $S$, where $p(X, Y)=1 / \alpha(U, V), q=\beta / \alpha$. From (13.9) we obtain

$$
p_{j k} U_{X}^{k}=V_{j Y}, \quad p_{j k} U_{Y}^{k}=-V_{j X} \quad \text { (p.p. on } D \text { ), }
$$


where $U^{1}=U, U^{2}=V, V_{1}=V, V_{2}=-U$ and

$$
p_{11}=\frac{p^{2}+q^{2}}{p}=\delta, \quad p_{12}=p_{21}=-\frac{q}{p}=-\beta, \quad p_{22}=\frac{1}{p}=\alpha .
$$

The functions $U, V$ are A.C.T. on $S[(13.3)]$. In addition, since $\alpha, \beta, \delta$ $\in C^{\prime}(S)$, the $p_{j k}$ are A.C.T. on $S[(13.7)$ and (13.11)]. Thus, from (13.10) and $p_{11} p_{22}-p_{12}^{2}=1$ on $S$ we see that $U^{i}, V_{j}$ satisfy the conditions of Theorem 9 , except possibly for (7.4). To show that (7.4) holds, let $t$ be fixed, $0<t<a$. Put $S_{t}=\sigma(t, 0)$. On the closed subset $\Gamma^{-1}\left(S_{t}^{c}\right)$ of $D$, we have $|\nabla \alpha|^{2},|\nabla \beta|^{2}$, $|\nabla \delta|^{2}, \alpha, \delta \leqq A$ where $A$ is independent of $z$. Using (13.7), (13.9), and (13.11), we obtain

$$
\begin{aligned}
\left|\nabla p_{11}\right|^{2} & \leqq|\nabla \delta|^{2}\left(|\nabla U|^{2}+|\nabla V|^{2}\right)=|\nabla \delta|^{2}(\alpha+\delta)\left(U_{X} V_{Y}-U_{Y} V_{X}\right) \\
& \leqq A\left(U_{X} V_{Y}-U_{Y} V_{X}\right)
\end{aligned}
$$

p.p. in $S_{t}^{c}$, where $A$ does not depend on $Z$. This with (13.5) gives for $Z_{0}$ on $S_{t}^{c}$

$$
\begin{aligned}
\left|\iint_{\sigma\left(h, Z_{0}\right) \cdot S t^{c}}\right| \nabla p_{11}|d S|^{2} & \leqq A h^{2} \iint_{\sigma\left(h, Z_{0}\right) \cdot S t^{c}}\left|\nabla p_{11}\right|^{2} d S \\
& \leqq A h^{2} \iint_{\sigma\left(h, Z_{0}\right) \cdot S t^{c}}\left(U_{X} V_{Y}-U_{Y} V_{X}\right) d S \\
& \leqq A h^{2} \operatorname{meas} \Gamma^{-1}\left[\left(\sigma\left(h, Z_{0}\right) \cdot S_{t}^{c}\right)\right],
\end{aligned}
$$

where $A$ does not depend on $h$ or $Z_{0}$. Now, by (13.6), we have

$$
\text { meas } \Gamma^{-1}\left(\sigma\left(h, Z_{0}\right) \cdot S_{t}^{c}\right) \leqq A h^{2 \tau}
$$

where $\tau=\tau(t)$, and $A$ is independent of $Z_{0},\left|Z_{0}\right| \leqq t$. We find then

$$
\iint_{\sigma\left(h, Z_{0}\right) \cdot S t^{c}}\left|\nabla p_{11}\right| d S \leqq A h^{1+\tau} \quad\left(\left|Z_{0}\right| \leqq t, 0<h\right),
$$

where $A$ does not depend on $h$ or $Z_{0}$. In similar manner we find that $p_{12}, p_{22}$ satisfy (13.12). Thus (7.4) is satisfied. It then follows from Theorem 9 that $U, V \in C^{\prime}$ on $S$. But now with $U, V \in C^{\prime}$ on $S$, we have $p, q \in C^{\prime}(S)$. Hence using Theorem 14 and (13.9) we find that $U_{X} V_{Y}-U_{Y} V_{X}>0$ on $S$. We conclude that $u, v \in C^{\prime}(D)$ and $u_{x} v_{y}-u_{y} v_{x}>0$ in $D$, which completes the proof.

14. Proof of Theorem I. On the basis of Theorems II and III, we can prove Theorem I without difficulty. Under the hypotheses of Theorem I, put $K=\left[\alpha \delta-(\beta+\gamma)^{2} / 4\right]^{1 / 2}$. Let $\zeta^{(1)}, \zeta^{(2)}, \zeta^{(3)}$ be distinct points on $S^{*}$, similar in order to $z^{(1)}, z^{(2)}, z^{(3)}$ on $D^{*}$. We first consider the equations

$$
\frac{\alpha}{K} \xi_{x}+\frac{\beta+\gamma}{2 K} \xi_{y}=\eta_{y}, \quad \frac{\beta+\gamma}{2 K} \xi_{x}+\frac{\delta}{K} \xi_{y}=-\eta_{x} .
$$


The coefficients in these equations satisfy the conditions prescribed in Theorem III in $D$. Hence there is a mapping $\xi=\xi(x, y), \eta=\eta(x, y)$, with inverse $x=x(\xi, \eta), y=y(\xi, \eta)$, of $D^{c}$ into $S^{c}$, satisfying the conclusions of that theorem with reference to $\zeta^{(1)}, \zeta^{(2)}, \zeta^{(3)}$ and the equations (14.1). We next consider the equations

$$
K X_{\xi}+\frac{\beta-\gamma}{2} X_{\eta}=Y_{\eta}, \quad \frac{\gamma-\beta}{2} X_{\xi}+K X_{\eta}=-Y_{\xi}
$$

where the coefficients are evaluated at $x=x(\xi, \eta), y=y(\xi, \eta)$. The coefficients here satisfy the conditions prescribed in Theorem II in $S$. Let $X=X(\xi, \eta)$, $Y=Y(\xi, \eta)$ be a mapping from $S^{c}$ into $T^{c}$ satisfying the conclusions of Theorem II with reference to $\zeta^{(1)}, \zeta^{(2)}, \zeta^{(8)}$, and the equations (14.2). We find then that

$$
u(x, y)=X(\xi(x, y), \eta(x, y)), \quad v(x, y)=Y(\xi, \eta)
$$

give the mapping whose existence was asserted in Theorem I.

\section{BiBLIOGRAPHY}

1. S. Agmon and L. Bers, The expansion theorem for pseudo-analytic functions, Proc. Amer. Math. Soc. vol. 3 (1952) pp. 757-767.

2. L. Bers, Partial differential equations and generalized analytic functions, Proc. Nat. Acad. Sci. U.S.A. vol. 36 (1950) pp. 130-136. Second note, ibid. vol. 37 (1951) pp. 42-47.

3. Jesse Douglas, Solution of the problem of Plateau, Trans. Amer. Math. Soc. vol. 33 (1931) pp. 263-321. 49.

4. G. C. Evans, Complements of potential theory, II, Amer. J. Math. vol. 55 (1933) pp. 29-

5. —, Fundamental points of potential theory, Rice Institute Pamphlets, vol. 7, No. 4 (1920) pp. 252-329.

6. J. J. Gergen and F. G. Dressel, Mapping by p-regular functions, Duke J. Math. vol. 18 (1951) pp. 185-210.

7. - Uniqueness for p-regular mapping, Duke Math J. vol. 19 (1952) pp. 435-444.

8. E. Goursat, Cours d'analyse mathématique, Paris, vol. 3, 1923.

9. Oliver Dimon Kellogg, Foundations of potential theory, Berlin, 1929.

10. Charles B. Morrey, Jr., On the solutions of quasilinear elliptic partial differential equations, Trans. Amer. Math. Soc. vol. 43 (1938) pp. 126-166.

11. L. Tonelli, Sulla quadratura della superficie, Atti della Reale Accademia dei Lincei (6) vol. 3 (1926) pp. 357-362, 445-450, 633-638, 714-719.

DURE UNIVERSITY,

DURHAM, N. C. 